I NTER NATIONAL MONETARY FUND

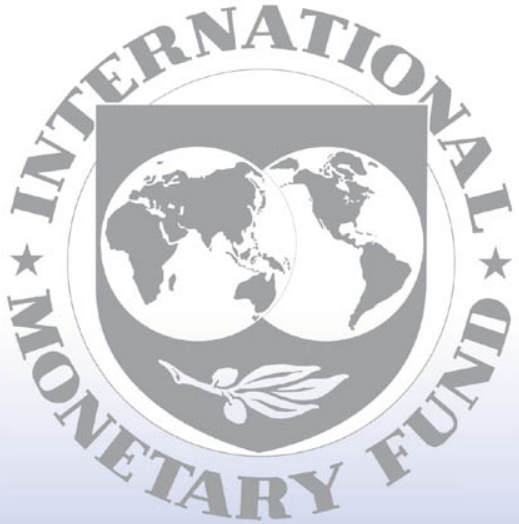

Staff

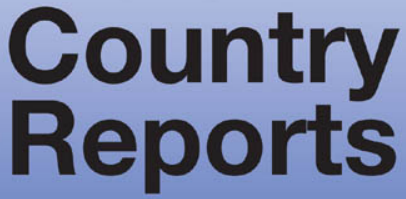




\title{
Algeria: Statistical Appendix
}

This statistical appendix on Algeria was prepared by a staff team of the International Monetary Fund as background documentation for the periodic consultation with the member country. It is based on the information available at the time it was completed on December 22, 2011. The views expressed in this document are those of the staff team and do not necessarily reflect the views of the government of Algeria or the Executive Board of the IMF.

The policy of publication of staff reports and other documents by the IMF allows for the deletion of market-sensitive information.

\author{
Copies of this report are available to the public from \\ International Monetary Fund • Publication Services \\ $70019^{\text {th }}$ Street, N.W. • Washington, D.C. 20431 \\ Telephone: (202) 623-7430 • Telefax: (202) 623-7201 \\ E-mail: publications@imf.org Internet: http://www.imf.org
}

\section{International Monetary Fund \\ Washington, D.C.}


This page intentionally left blank

CInternational Monetary Fund. Not for Redistribution 


\title{
INTERNATIONAL MONETARY FUND
}

\author{
ALGERIA
}

\section{Statistical Appendix}

Prepared by Jos Gij n (MCD)

Approved by the Middle East and Central Asia Department

December 22, 2011

Contents

Tables

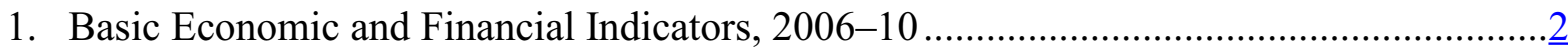

2. Supply and Use of Resources at Current Prices, 2006-10 .............................................

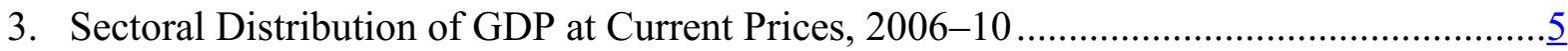

4. Sectoral Distribution of Real GDP Growth, 2006-10 …....................................................

5. Production, Exports, and Consumption of Liquid Petroleum Products, 2006-10 …........... 1

6. Production, Exports, and Consumption of Gas Products, 2006-10 ……………............. $\underline{8}$

7. Domestic Prices of Major Energy Products, 2006-10 …..............................................

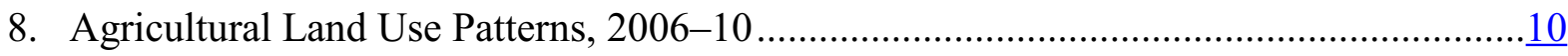

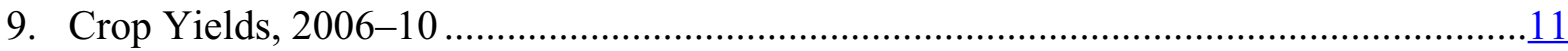

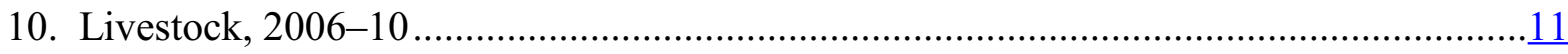

11. Indices of Industrial Production in Public Enterprises, 2006-10 .................................

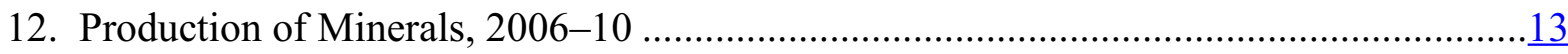

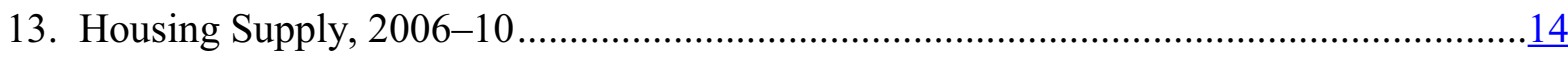

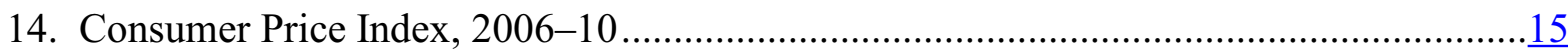

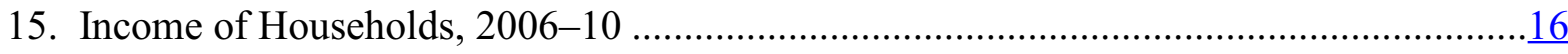

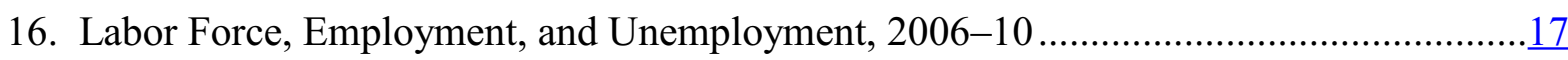

17. Summary of Central Government Operations, 2006-10 ............................................18

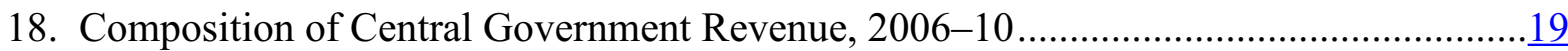

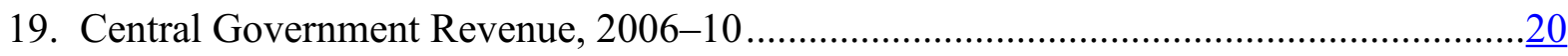

20. Composition of Central Government Expenditure, 2006-10 …......................................

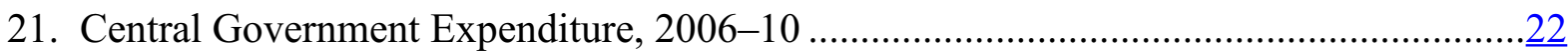

22. Sectoral Allocation Budgetary Capital Expenditure, 2006-10 ….....................................

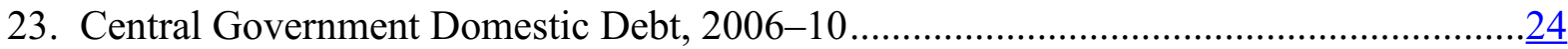

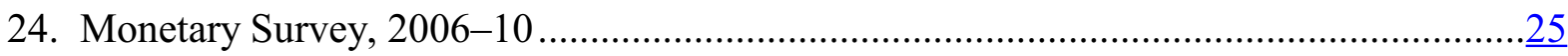

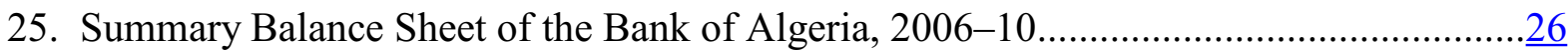

26. Summary Balance Sheet of Deposit Money Banks, 2006-10 ………………................27 


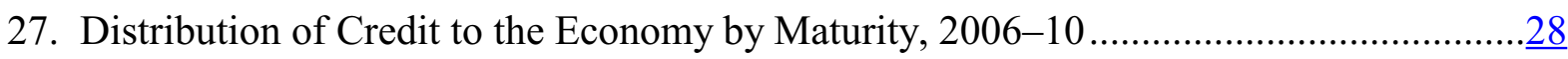

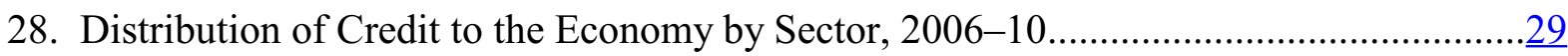

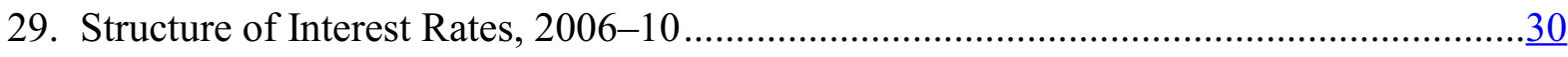

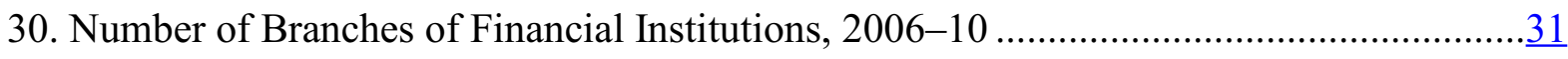

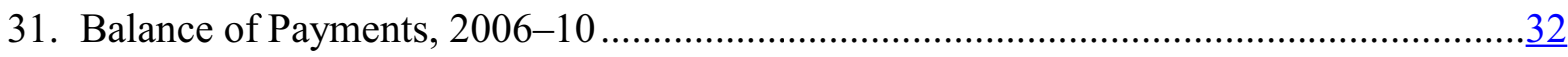

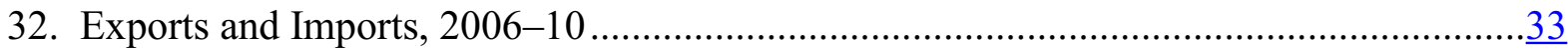

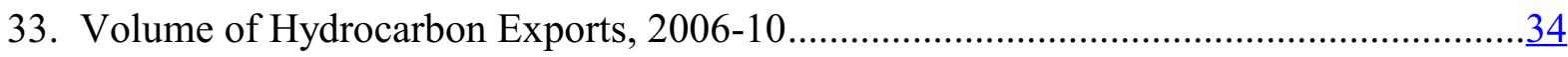

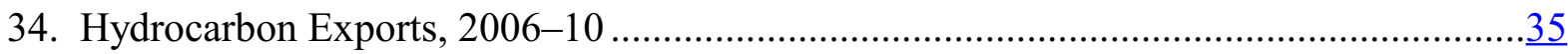

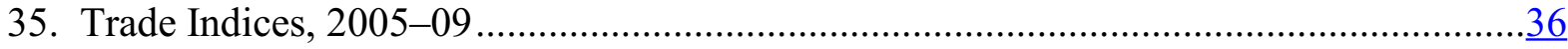

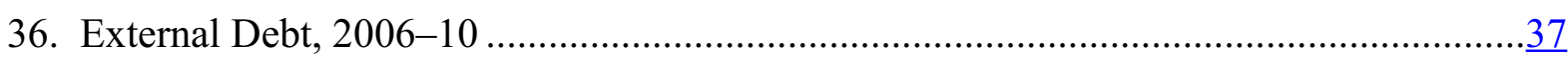

37. Nominal and Real Effective Exchange Rates, 1990-2010 ........................................... 
Table 1. Algeria: Basic Economic and Financial Indicators, 2006-10

\begin{tabular}{|c|c|c|c|c|c|}
\hline & 2006 & 2007 & 2008 & 2009 & 2010 \\
\hline & \multicolumn{5}{|c|}{ (Annual percentage change) } \\
\hline \multicolumn{6}{|l|}{ Production and prices } \\
\hline Nominal GDP & 12.6 & 10.0 & 18.4 & -9.5 & 20.1 \\
\hline Real GDP & 2.0 & 3.0 & 2.4 & 2.4 & 3.3 \\
\hline Real nonhydrocarbon GDP (NHGDP) & 5.6 & 6.3 & 6.1 & 9.3 & 5.9 \\
\hline GDP deflator & 10.3 & 7.3 & 15.4 & -9.9 & 16.3 \\
\hline NHGDP deflator & 4.1 & 8.1 & 8.3 & 4.2 & 9.3 \\
\hline \multirow[t]{2}{*}{ Consumer price index (average) } & 2.5 & 3.5 & 4.5 & 5.7 & 3.9 \\
\hline & \multicolumn{5}{|c|}{ (In billions of U.S. dollars; unless otherwise indicated) } \\
\hline \multicolumn{6}{|l|}{ External sector } \\
\hline Exports, f.o.b. & 54.7 & 60.6 & 78.6 & 45.2 & 57.1 \\
\hline Imports, f.o.b. & 20.7 & 26.4 & 38.0 & 37.4 & 38.9 \\
\hline Current account balance & 29.0 & 30.6 & 34.5 & 0.4 & 12.2 \\
\hline Current account balance (in percent of GDP) & 24.7 & 22.5 & 20.1 & 0.3 & 7.6 \\
\hline \multirow[t]{2}{*}{ Overall balance } & 17.7 & 29.6 & 37.0 & 3.9 & 15.6 \\
\hline & \multicolumn{5}{|c|}{ (In percent of GDP; unless otherwise indicated) } \\
\hline \multicolumn{6}{|l|}{ Central government } \\
\hline Total budget revenue and grants & 42.8 & 39.2 & 47.2 & 36.3 & 36.4 \\
\hline Of which: hydrocarbon revenue & 32.9 & 30.1 & 37.2 & 23.8 & 24.1 \\
\hline Total expenditure and net lending & 29.2 & 34.7 & 39.5 & 43.1 & 38.8 \\
\hline Overall balance & 13.5 & 4.4 & 7.7 & -6.4 & -2.4 \\
\hline Nonhydrocarbon primary balance (in percent of NHGDP) & -34.1 & -43.2 & -53.1 & -44.9 & -40.2 \\
\hline \multicolumn{6}{|l|}{ Money and credit } \\
\hline Broad money (annual percentage change) & 18.6 & 24.2 & 16.1 & 3.1 & 13.8 \\
\hline Net credit to government (as a percent of beginning money stock & -17.8 & -20.7 & -25.2 & 0.2 & -4.4 \\
\hline Credit to the economy (annual percentage change) & 9.3 & 12.5 & 20.4 & 18.5 & 5.1 \\
\hline 13-week T-bill interest rate (in percent per annum) & 1.4 & 0.2 & 0.2 & 0.3 & 0.2 \\
\hline \multicolumn{6}{|l|}{ Official reserves } \\
\hline Gross official reserves (in billions of U.S. dollars) & 77.8 & 110.2 & 143.1 & 148.9 & 162.2 \\
\hline Idem, in months of next year's imports of goods and services & 28.0 & 27.4 & 35.4 & 35.6 & 38.3 \\
\hline \multicolumn{6}{|l|}{ Debt } \\
\hline Total external debt (in billions of U.S. dollars) & 5.6 & 5.6 & 5.6 & 5.7 & 5.7 \\
\hline Total external debt (in percent of GDP) & 4.8 & 4.2 & 3.3 & 3.8 & 3.5 \\
\hline Total government debt (in percent of GDP) & 23.6 & 12.4 & 8.2 & 10.9 & 10.0 \\
\hline Domestic government debt (in percent of GDP) & 18.7 & 5.3 & 4.9 & 7.4 & 6.2 \\
\hline \multicolumn{6}{|l|}{ Memorandum items: } \\
\hline Gross domestic product (in billions of dinars) & 8,515 & 9,367 & 11,090 & 10,034 & 12,050 \\
\hline Idem (in billions of U.S. dollars) & 116.8 & 135.3 & 171.0 & 139.8 & 160.8 \\
\hline Per capita GDP (in U.S. dollars) & 3,456 & 3,934 & 4,962 & $3,925.9$ & $4,516.3$ \\
\hline Exchange rate (DA/US\$) (average) & 73.7 & 69.2 & 64.6 & 72.5 & 74.9 \\
\hline Real effective exchange rate (annual percentage rate) & -0.2 & -1.4 & 4.9 & -1.2 & 0.4 \\
\hline Terms of trade (annual percentage rate) & 9.6 & 0.8 & 22.5 & -26.8 & 22.9 \\
\hline
\end{tabular}

Sources: Algerian authorities; and Fund staff estimates. 
Table 2. Algeria: Supply and Use of Resources at Current Prices, 2006-10

\begin{tabular}{|c|c|c|c|c|c|}
\hline & 2006 & 2007 & 2008 & 2009 & 2010 \\
\hline & \multicolumn{5}{|c|}{ (In billions of dinars) } \\
\hline Gross domestic product & $8,514.8$ & $9,366.5$ & $11,090.0$ & $10,034.3$ & $12,049.5$ \\
\hline Resource gap & $2,286.2$ & $2,076.1$ & $2,127.3$ & -57.9 & 861.5 \\
\hline Exports of goods and nonfactor services & $4,149.7$ & $4,402.2$ & $5,298.1$ & $3,525.9$ & $4,610.1$ \\
\hline Imports of goods and nonfactor services & $1,863.5$ & $2,326.1$ & $3,170.8$ & $3,583.8$ & $3,748.6$ \\
\hline Gross domestic spending & $6,234.4$ & $7,290.4$ & $8,962.7$ & $10,092.2$ & $11,188.0$ \\
\hline Consumption & $3,650.4$ & $4,052.8$ & $4,805.7$ & $5,414.7$ & $6,322.2$ \\
\hline Government & 954.9 & $1,089.0$ & $1,459.1$ & $1,646.2$ & $2,166.9$ \\
\hline Nongovernment & $2,695.6$ & $2,963.8$ & $3,346.6$ & $3,768.5$ & $4,155.3$ \\
\hline Gross investment & $2,583.9$ & $3,237.6$ & $4,157.0$ & $4,677.5$ & $4,865.80$ \\
\hline Gross fixed capital formation & $1,967.3$ & $2,462.1$ & $3,228.3$ & $3,811.4$ & $4,350.9$ \\
\hline Change in inventories & 616.6 & 775.5 & 928.7 & 866.1 & 514.9 \\
\hline Gross domestic savings & $4,864.4$ & $5,313.7$ & $6,284.3$ & $4,619.6$ & $5,727.3$ \\
\hline Net factor income from abroad & -328.9 & -127.6 & -84.2 & -94.8 & -26.4 \\
\hline Net current transfers & 116.7 & 153.3 & 175.1 & 191.3 & 197.1 \\
\hline National savings & $4,652.2$ & $5,339.4$ & $6,375.2$ & $4,716.1$ & 5898 \\
\hline \multirow[t]{2}{*}{ Gross national product } & $8,185.9$ & $9,238.9$ & $11,005.8$ & $9,939.5$ & $12,023.1$ \\
\hline & \multicolumn{4}{|c|}{ (In percent of GDP) } & \\
\hline Gross domestic product & 100.0 & 100.0 & 100.0 & 100.0 & 100.0 \\
\hline Resource gap & 26.8 & 22.2 & 19.2 & -0.6 & 7.1 \\
\hline Exports of goods and nonfactor services & 48.7 & 47.0 & 47.8 & 35.1 & 38.3 \\
\hline Imports of goods and nonfactor services & 21.9 & 24.8 & 28.6 & 35.7 & 31.1 \\
\hline Gross domestic spending & 73.2 & 77.8 & 80.8 & 100.6 & 92.9 \\
\hline Consumption & 42.9 & 43.3 & 43.3 & 54.0 & 52.5 \\
\hline Government & 11.2 & 11.6 & 13.2 & 16.4 & 18.0 \\
\hline Nongovernment & 31.7 & 31.6 & 30.2 & 37.6 & 34.5 \\
\hline Gross investment & 30.3 & 34.6 & 37.5 & 46.6 & 40.4 \\
\hline Gross fixed capital formation & 23.1 & 26.3 & 29.1 & 38.0 & 36.1 \\
\hline Change in inventories & 7.2 & 8.3 & 8.4 & 8.6 & 4.3 \\
\hline Gross domestic savings & 57.1 & 56.7 & 56.7 & 46.0 & 47.5 \\
\hline Net factor income from abroad & -3.9 & -1.4 & -0.8 & -0.9 & -0.2 \\
\hline Net current transfers & 1.4 & 1.6 & 1.6 & 1.9 & 1.6 \\
\hline National savings & 54.6 & 57.0 & 57.5 & 47.0 & 48.9 \\
\hline Gross national product & 96.1 & 98.6 & 99.2 & 99.1 & 99.8 \\
\hline
\end{tabular}

Sources: Algerian authorities; and Fund staff estimates. 
Table 3. Algeria: Sectoral Distribution of GDP at Current Prices, 2006-10

\begin{tabular}{|c|c|c|c|c|c|}
\hline & 2006 & 2007 & 2008 & 2009 & 2010 \\
\hline & \multicolumn{5}{|c|}{ (In billions of dinars) } \\
\hline Hydrocarbons & $3,882.2$ & $4,089.3$ & $4,997.6$ & $3,109.1$ & $4,180.4$ \\
\hline Other sectors & $4,141.1$ & $4,744.9$ & $5,438.5$ & $6,209.4$ & $7,130.0$ \\
\hline Agriculture & 641.3 & 708.1 & 727.4 & 931.3 & $1,015.2$ \\
\hline Industry & 449.5 & 479.8 & 519.5 & 573.1 & 597.9 \\
\hline Construction and public works & 674.3 & 825.1 & 956.7 & $1,094.8$ & $1,257.4$ \\
\hline Nongovernment services & $1,698.1$ & $1,933.2$ & $2,147.0$ & $2,384.6$ & $2,638.7$ \\
\hline Government services & 677.9 & 798.7 & $1,087.9$ & $1,225.6$ & $1,620.8$ \\
\hline Imports taxes and duties & 491.5 & 532.4 & 653.9 & 715.8 & 739.1 \\
\hline \multirow[t]{2}{*}{ Gross domestic product } & $8,514.8$ & $9,366.6$ & $11,090.0$ & $10,034.3$ & $12,049.5$ \\
\hline & \multicolumn{5}{|c|}{ (In percent of GDP) } \\
\hline Hydrocarbons & 45.6 & 43.7 & 45.1 & 31.0 & 34.7 \\
\hline Other sectors & 48.6 & 50.7 & 49.0 & 61.9 & 59.2 \\
\hline \multicolumn{6}{|l|}{ Of which: } \\
\hline Agriculture & 7.5 & 7.6 & 6.6 & 9.3 & 8.4 \\
\hline Industry & 5.3 & 5.1 & 4.7 & 5.7 & 5.0 \\
\hline \multirow[t]{2}{*}{ Government services } & 8.0 & 8.5 & 9.8 & 12.2 & 13.5 \\
\hline & \multicolumn{5}{|c|}{ (Annual percentage change) } \\
\hline Hydrocarbons & 15.8 & 5.3 & 22.2 & -37.8 & 34.5 \\
\hline Other sectors & 11.4 & 14.6 & 14.6 & 14.2 & 14.8 \\
\hline \multicolumn{6}{|l|}{ Of which: } \\
\hline Agriculture & 10.3 & 10.4 & 2.7 & 28.0 & 9.0 \\
\hline Industry & 6.8 & 6.7 & 8.3 & 10.3 & 4.3 \\
\hline Government services & 7.3 & 17.8 & 36.2 & 12.7 & 32.2 \\
\hline Gross domestic product & 12.6 & 10.0 & 18.4 & -9.5 & 20.1 \\
\hline
\end{tabular}

Sources: Algerian authorities; and Fund staff estimates. 
Table 4. Algeria: Sectoral Distribution of Real GDP Growth, 2006-10

(In percent)

\begin{tabular}{|c|c|c|c|c|c|}
\hline & 2006 & 2007 & 2008 & 2009 & 2010 \\
\hline Hydrocarbons & -2.5 & -0.9 & -2.3 & -6.0 & -2.6 \\
\hline \multicolumn{6}{|l|}{ Other sectors } \\
\hline Agriculture & 4.9 & 5.0 & -5.3 & 20 & 6 \\
\hline Mining & 15.1 & 8 & 9.8 & 3.4 & -3.3 \\
\hline Energy (nonhydroelectric) and water & 3.4 & 6.0 & 7.9 & 7.2 & 5.6 \\
\hline Manufacturing & -2.2 & -3.9 & 1.9 & 0.7 & -2.5 \\
\hline Food processing & -7.6 & -2.1 & 6.8 & -9.1 & -3.3 \\
\hline Steel, mechanical, and electrical construction & 1.3 & -8.9 & 3.9 & 6.4 & -12.6 \\
\hline Chemical industry & -3.1 & -4.4 & 2.5 & 2.0 & -10.6 \\
\hline Textiles & -14.0 & -15 & -1.1 & 1.2 & -10.8 \\
\hline Leather products & -15.9 & -4.8 & -1.2 & -12.0 & -6.3 \\
\hline Building materials & -3.8 & 2.7 & -1.6 & -1.0 & -5 \\
\hline Wood and paper & 2.9 & -6.8 & -11.9 & -21.4 & 14.8 \\
\hline Other & -1.0 & -31.2 & -20.6 & 0.0 & -21.3 \\
\hline Private industry & 2.1 & 3.2 & $\ldots$ & $\ldots$ & \\
\hline Construction and public works & 11.6 & 9.8 & 9.8 & 8.7 & 6.6 \\
\hline Nongovernment services & 6.5 & 6.8 & 7.8 & 8.8 & 6.9 \\
\hline Government services & 3.1 & 6.5 & 8.4 & 7.0 & 6 \\
\hline Import taxes and duties & 2.7 & 5.5 & 7.7 & 6.8 & 5.8 \\
\hline Gross domestic product & 2.0 & 3.0 & 2.4 & 2.4 & 3.3 \\
\hline Gross domestic absorption & 3.6 & 7.9 & 10.4 & 6.9 & 5.5 \\
\hline Consumption & 3.4 & 3.7 & 5.5 & 5.8 & 5.8 \\
\hline Government & 4.8 & 7.1 & 8.7 & 6.0 & 6.2 \\
\hline Nongovernment & 2.9 & 2.5 & 4.9 & 5.8 & 5.6 \\
\hline Gross investment & 7.2 & 9.8 & 11.8 & 8.5 & 5.2 \\
\hline Exports of goods and nonfactor services & -2.5 & -0.6 & -2.9 & -10.7 & -1.7 \\
\hline Imports of goods and nonfactor services & -1.8 & 11.1 & 17.7 & 15.6 & 2.4 \\
\hline \multicolumn{6}{|l|}{ Memorandum item: } \\
\hline Real per capita GDP & -0.7 & 1.2 & 2.2 & 0.7 & 1.8 \\
\hline
\end{tabular}

Source: Algerian authorities. 
Table 5. Algeria: Production, Exports, and Consumption of Liquid Petroleum Products, 2006-10

(In millions of tons)

\begin{tabular}{lrrrrr}
\hline & 2006 & 2007 & 2008 & 2009 & 2010 \\
\hline Liquid petroleum and substitutes & & & & & \\
Production & 85.6 & 85.8 & 83.4 & 77.2 & 73.7 \\
$\quad$ Crude oil and substitutes & 63.7 & 63.8 & 61.3 & 56.8 & 55.3 \\
Condensate & 13.6 & 13.7 & 13.5 & 12.3 & 11.2 \\
$\quad$ Liquefied petroleum gas (LPG) & 8.3 & 8.3 & 8.6 & 8.1 & 7.2 \\
Imports & 0.3 & 0.4 & 0.4 & 0.4 & 0.3 \\
Refinery input & 20.1 & 19.5 & 22.3 & 23.7 & 27.3 \\
Direct exports & 64.2 & 63.5 & 59.7 & 97.2 & 45 \\
$\quad$ Crude & 43.8 & 43.2 & 39.2 & 52 & 33 \\
Condensate & 13.4 & 12.9 & 13.0 & 34.7 & 6.1 \\
LPG & 7.0 & 7.4 & 7.5 & 10.5 & 5.9 \\
LPG consumption & 1.9 & 1.9 & 2.0 & 6.8 & 1.9 \\
Discrepancy 1/ & -0.3 & 1.3 & -0.2 & 2.0 & -0.2 \\
Refined products & & & & & \\
Production & 19.0 & 19.1 & 21.4 & 22.4 & 26.1 \\
Exports & 9.6 & 9.9 & 10.4 & 10.8 & 14.1 \\
Domestic consumption & 11.4 & 12.2 & 13.4 & 14.3 & 14.2 \\
Discrepancy 1/ & -2.0 & -3.0 & -2.4 & -2.7 & -2.2 \\
Memorandum item: & & & & & \\
Total exports & 73.8 & 73.5 & 70.1 & 62.9 & 59.1 \\
\hline
\end{tabular}

Source: Algerian authorities.

1/ Reflects change in inventories and errors of measurement. 
Table 6. Algeria: Production, Exports, and Consumption of Gas Products, 2006-10

\begin{tabular}{|c|c|c|c|c|c|}
\hline & 2006 & 2007 & 2008 & 2009 & 2010 \\
\hline & \multicolumn{5}{|c|}{ (In billions of cubic meters) } \\
\hline Gross production & 194.8 & 198.2 & 201.5 & 196.9 & 192.1 \\
\hline Input into oil production & 84.5 & 84.8 & 85.1 & 79.5 & 80.4 \\
\hline Net production $1 /$ & 110.3 & 113.4 & 116.4 & 117.4 & 111.7 \\
\hline Volume transported 2/ & 84.9 & 85.4 & 86.2 & 82 & 83.5 \\
\hline Domestic consumption & 17.0 & 17.6 & 19.4 & 20.4 & 23 \\
\hline Sales to liquefaction plants & 30.2 & 30.5 & 26.7 & 27.1 & 23.9 \\
\hline $\begin{array}{l}\text { Liquid natural gas (LNG) productior } \\
\text { Of which: }\end{array}$ & 23.5 & 24 & 21.9 & 22.1 & 19.5 \\
\hline LNG exports & 23.2 & 23.5 & 21.7 & 21.8 & 19.5 \\
\hline Exports by pipeline & 37.8 & 35.5 & 39 & 33.6 & 37.8 \\
\hline \multirow[t]{2}{*}{ Discrepancy 3/ } & 0.0 & 0.0 & 0.0 & 0.0 & 0.0 \\
\hline & \multicolumn{5}{|c|}{ (Annual percentage change) } \\
\hline Gross production & 0.9 & 2.7 & 3.4 & -0.7 & -4.7 \\
\hline Input into oil production & -4.2 & -3.9 & 0.7 & -6.3 & -5.5 \\
\hline Net production 1/ & 5.2 & 8.2 & 5.5 & 3.5 & -4.0 \\
\hline Volume transported 2/ & -4.8 & -4.3 & 1.5 & -4.0 & -3.1 \\
\hline Domestic consumption & 4.9 & 8.6 & 14.1 & 15.9 & 18.6 \\
\hline Sales to liquefaction plants & -2.3 & -1.3 & -11.6 & -11.1 & -10.5 \\
\hline LNG production & -4.9 & -2.8 & -6.8 & -7.9 & -11.0 \\
\hline \multicolumn{6}{|l|}{ Of which: } \\
\hline LNG exports & -4.9 & -3.7 & -6.5 & -7.2 & -10.1 \\
\hline \multirow[t]{2}{*}{ Exports by pipeline } & -5.0 & -10.8 & 3.2 & -5.4 & -3.1 \\
\hline & \multicolumn{5}{|c|}{ (In billions of cubic meters; unless specified otherwise) } \\
\hline \multicolumn{6}{|l|}{ Memorandum item: } \\
\hline Total exports & 62.5 & 58.9 & 60.6 & 55.4 & 57.4 \\
\hline \multicolumn{6}{|l|}{ Of which: } \\
\hline LNG exports (in percent) & 37.1 & 39.9 & 35.7 & 39.4 & 34.0 \\
\hline Piped exports (in percent) & 60.6 & 60.3 & 64.3 & 60.6 & 66.0 \\
\hline
\end{tabular}

Source: Algerian authorities.

$1 /$ Net of gas reinjected into producing oil wells.

2/ Equal to net production minus gas flared, gas used for lifting and for fuel gas, and other losses in the fields.

3/ Reflects errors in measurement. 
Table 7. Algeria: Domestic Prices of Major Energy Products, 2006-10 (In dinars per liter; unless otherwise indicated)

\begin{tabular}{lrrrrr}
\hline & 2006 & 2007 & 2008 & 2009 & 2010 \\
\hline Butane (13 kg/bottle) & 200.0 & 200.0 & 200.0 & 200.0 & 200.0 \\
Propane (35 kg/bottle) & 400.0 & 400.0 & 400.0 & 400.0 & 400.0 \\
LPG fuel (kg) & 5.9 & 5.9 & 5.9 & 5.9 & 5.9 \\
LPG car fuel & 9.0 & 9.0 & 9.0 & 9.0 & \\
Super gasoline & 23.0 & 23.0 & 23.0 & 23.0 & 23.0 \\
Regular gasoline & 21.2 & 21.2 & 21.2 & 21.2 & 21.2 \\
Gas oil & 13.7 & 13.7 & 13.7 & 13.7 & 13.7 \\
Fuel oil & 10.0 & 10.0 & 10.0 & 10.0 & 10.0 \\
\hline
\end{tabular}

Source: Algerian authorities. 
Table 8. Algeria: Agricultural Land Use Patterns, 2006-10

(In thousands of hectares)

\begin{tabular}{lrrrrr}
\hline & 2006 & 2007 & 2008 & 2009 & 2010 \\
\hline Cereals & $3,266.7$ & $3,056.5$ & $3,056.4$ & 3276.2 & 3327.3 \\
$\quad$ Durum wheat & $1,358.0$ & $1,250.6$ & $1,230.3$ & 1288.3 & 1339.4 \\
$\quad$ Bread wheat & 700.1 & 661.1 & 541.5 & 607.2 & 607.2 \\
Barley & $1,117.7$ & $1,057.7$ & $1,195.3$ & 1296.6 & 1296.6 \\
$\quad$ Other & 90.9 & 87.1 & 89.3 & 84.1 & 84.1 \\
Pulses & 66.9 & 63.5 & 61.2 & 67.4 & 74.2 \\
Fodder crops & 364.7 & 493.8 & 588.9 & 712.6 & 893.6 \\
Industrial crops 1/ & 15.7 & 15.7 & 22.4 & 21.2 & 25.4 \\
Vegetables 2/ & 212.4 & 193.6 & 205.6 & 348.8 & 245.8 \\
Grapes & 97.0 & 91.9 & 86.7 & 82.7 & 80.4 \\
Fruit trees & 810.1 & 828.0 & 823.8 & 823.2 & 828.4 \\
Natural prairies & 25.6 & 25.5 & 24.3 & 24.5 & 24.5 \\
Others & $3,544.5$ & $3,573.0$ & $3,563.3$ & $\ldots$ & $\ldots$ \\
Total cultivated land & $8,403.6$ & $8,414.7$ & $8,424.8$ & $8,423.3$ & $8,435.0$ \\
Fallow & $3,404.8$ & $3,573.0$ & $3,563.3$ & $3,423.5$ & $3,275.7$ \\
\hline
\end{tabular}

Source: Algerian authorities.

$1 /$ Industrial tomatoes and tobacco.

2/ Potatoes, tomatoes, garlic and onions, and watermelons. 
Table 9. Algeria: Crop Yields, 2006-10

(In thousands of quintals)

\begin{tabular}{lrrrrr}
\hline & 2006 & 2007 & 2008 & 2009 & 2010 \\
\hline Cereals & & & & & \\
$\quad$ Hard wheat & $17,728.0$ & $15,290.0$ & $9,350.0$ & $20,010.4$ & $20,385.0$ \\
$\quad$ Soft wheat & $9,151.3$ & $7,899.6$ & $3,437.0$ & $9,520.8$ & $9,142.0$ \\
$\quad$ Barley & $12,358.8$ & $11,866.6$ & $3,873.0$ & $22,033.6$ & $16,054.0$ \\
Potatoes & $21,809.6$ & $15,068.6$ & $21,710.6$ & $26,360.6$ & $33,003.1$ \\
Pulses & & & & & \\
$\quad$ Fava beans & 243.0 & 279.7 & 235.2 & 364.9 & 366.3 \\
$\quad$ Chickpeas & 127.1 & 142.9 & 112.1 & 178.4 & 234.7 \\
\hline
\end{tabular}

Source: Algerian authorities.

Table 10. Algeria: Livestock, 2006-10

(In thousands of heads)

\begin{tabular}{lrrrrr}
\hline & 2006 & 2007 & 2008 & 2009 & 2010 \\
\hline Bovine & 1,608 & 1,658 & 1,670 & 1,682 & 1,747 \\
Ovine & 19,616 & 19,851 & 20,235 & 21,404 & 22,869 \\
Caprine & 3,755 & 3,774 & 3,829 & 3,962 & 4,287 \\
Cameline & 287 & 292 & 299 & 301 & 314 \\
\hline
\end{tabular}

Source: Algerian authorities. 
Table 11. Algeria: Indices of Industrial Production in Public Enterprises, 2006-10

\begin{tabular}{|c|c|c|c|c|c|}
\hline & 2006 & 2007 & 2008 & 2009 & 2010 \\
\hline & \multicolumn{5}{|c|}{$(2001=100)$} \\
\hline Water and energy & 239.6 & 253.9 & 273.9 & 293.4 & 309.8 \\
\hline Hydrocarbons & 148.5 & 150.7 & 147.1 & 139.2 & 136.3 \\
\hline Mining and quarrying & 104.8 & 120.9 & 132.8 & 137.3 & 132.8 \\
\hline Mechanical and electrical & 71.5 & 55.5 & 57.7 & 62.9 & 55 \\
\hline Construction materials & 108.5 & 116.1 & 114.2 & 113.1 & 107.4 \\
\hline Chemicals & 77.7 & 67.4 & 69.1 & 70.5 & 63 \\
\hline Food processing & 32.0 & 32.3 & 34.5 & 31.3 & 30.27 \\
\hline Textiles & 21.2 & 18.4 & 18.2 & 18.4 & 16.43 \\
\hline Leather and hides & 9.2 & 7.4 & 7.4 & 6.6 & 6.155 \\
\hline Woods and paper & 23.7 & 23.2 & 20.4 & 16.1 & 18.42 \\
\hline Other industries & 18.8 & 13 & 10.3 & 10.3 & 8.14 \\
\hline General index & 88.4 & 90.1 & 91.8 & 92.2 & 89.9 \\
\hline General index (excluding hydrocarbons) & 74.2 & 73.1 & 76.3 & 78.9 & 76.8 \\
\hline \multirow[t]{2}{*}{ General index of manufactured commodities } & 58.5 & 51.8 & 52.8 & 53.6 & 50.7 \\
\hline & \multicolumn{5}{|c|}{ (Annual percentage change) } \\
\hline Water and energy & 3.4 & 6.0 & 7.9 & 7.1 & 5.6 \\
\hline Hydrocarbons & -0.7 & 1.5 & -2.4 & -5.4 & -2.1 \\
\hline Mining and quarrying & 15.2 & 15.4 & 9.8 & 3.4 & -3.3 \\
\hline Mechanical and electrical & 1.3 & -22.4 & 4.0 & 9.0 & -12.6 \\
\hline Construction materials & -3.7 & 7.0 & -1.6 & -1.0 & -5.0 \\
\hline Chemicals & -5.6 & -13.3 & 2.5 & 2.0 & -10.6 \\
\hline Food processing & -7.5 & 0.9 & 6.8 & -9.3 & -3.3 \\
\hline Textiles & -13.8 & -13.2 & -1.1 & 1.2 & -10.8 \\
\hline Leather and hides & -15.6 & -19.6 & 0.0 & -10.8 & -6.7 \\
\hline Woods and paper & 2.6 & -2.1 & -12.1 & -21.3 & 14.8 \\
\hline Other industries & -1.1 & -30.9 & -20.8 & 0.4 & -21.3 \\
\hline General index & -0.3 & 1.9 & 1.9 & 0.4 & -2.5 \\
\hline General index (excluding hydrocarbons) & -0.5 & -1.5 & 4.4 & 3.4 & -2.7 \\
\hline General index of manufactured commodities & -2.5 & -11.5 & 1.9 & 1.5 & -5.4 \\
\hline
\end{tabular}

Source: Algerian authorities. 
Table 12. Algeria: Production of Minerals, 2006-10 1/

\begin{tabular}{lrrrrr}
\hline & 2006 & 2007 & 2008 & 2009 & 2010 \\
\hline & \multicolumn{3}{c}{$($ In thousands of tons; unless otherwise indicated) } \\
Iron ore & $1,996.0$ & $1,584.0$ & $2,068.0$ & $1,218.0$ & $1,014.0$ \\
Phosphates & $1,510.0$ & $1,800.0$ & $1,805.0$ & $1,017.0$ & $1,525.0$ \\
Zinc and lead & 0.6 & 0.0 & 0.0 & 0.0 & 0 \\
Mercury 2/ & 0.0 & 0.0 & 0.0 & 0.0 & 0 \\
& & $($ Annual percentage change) & \\
Iron ore & 19.7 & -20.6 & 30.6 & -41.1 & -16.7 \\
Phosphates & 73.2 & 19.2 & 0.3 & -43.7 & 50.0 \\
Zinc and lead & -85.8 & -100.0 & 0.0 & 0.0 & 0.0 \\
Mercury 2/ & 0.0 & 0.0 & 0.0 & 0.0 & 0.0 \\
\hline
\end{tabular}

Source: Algerian authorities.

1/ Excluding hydrocarbons.

2/ In thousands of containers, each weighing 34 kilograms. 
Table 13. Algeria: Housing Supply, 2006-10

\begin{tabular}{lrrrrr}
\hline & 2006 & 2007 & 2008 & 2009 & 2010 \\
\hline & \multicolumn{4}{c}{ (In thousands of dwellings delivered) } \\
Formal & 159.2 & 165.3 & 205.6 & 199.6 & 179.2 \\
Social & 150.7 & 160.3 & 201.5 & 194.0 & 174.3 \\
$\quad$ Rental & 43.5 & 44.1 & 57.6 & 55.5 & 61.3 \\
Owner occupied & 107.2 & 116.2 & 143.9 & 138.5 & 113.0 \\
Other formal & 8.4 & 5 & 4.1 & 5.6 & 4.9 \\
Public & 5.8 & 2.8 & 1.8 & 2 & 2.1 \\
Private & 2.6 & 2.2 & 2.3 & 3.6 & 2.8 \\
Autoconstruction & 18.6 & 14.7 & 15.1 & 18.2 & 11.7 \\
Total & 177.8 & 180 & 220.8 & 217.8 & 190.9 \\
& & $($ In percent of total) & & \\
Formal & & 91.8 & 93.1 & 91.6 & 93.9 \\
Social & 89.5 & 89.1 & 91.3 & 89.1 & 91.3 \\
$\quad$ Rental & 84.8 & 24.5 & 26.1 & 25.5 & 32.1 \\
Owner occupied & 24.5 & 64.6 & 65.2 & 63.6 & 59.2 \\
Other formal & 60.3 & 2.8 & 1.9 & 2.6 & 2.6 \\
$\quad$ Public & 4.7 & 1.6 & 0.8 & 0.9 & 1.1 \\
Private & 3.3 & 1.2 & 1.0 & 1.7 & 1.5 \\
Autoconstruction & 1.5 & 8.2 & 6.8 & 8.4 & 6.1 \\
Total & 10.5 & 100.0 & 100.0 & 100.0 \\
\hline
\end{tabular}

Source: Algerian authorities. 
Table 14. Consumer Price Index, 2006-10 1/

\begin{tabular}{|c|c|c|c|c|c|}
\hline & 2006 & 2007 & 2008 & $2009^{21}$ & 2010 \\
\hline & \multicolumn{5}{|c|}{$(2001=100)$} \\
\hline Foodstuffs, beverages, and tobacco & 675.9 & 720.2 & 773.4 & 139.0 & 144.9 \\
\hline Clothing and shoes & 444.5 & 442.9 & 440.4 & 104.6 & 107.5 \\
\hline Housing costs & 754.0 & 761.2 & 770.6 & 129.3 & 131.7 \\
\hline Furniture & 370.3 & 371 & 371.7 & 109.7 & 113.6 \\
\hline Health and medical care & 681.2 & 701.8 & 716.9 & 114.2 & 117.5 \\
\hline Transportation and communications & 670.2 & 667.1 & 672.2 & 141.8 & 145.3 \\
\hline Education and entertainment & 475.9 & 484.7 & 503.3 & 119.1 & 118.9 \\
\hline Other services & 741.3 & 754.1 & 780.7 & 127.8 & 141.8 \\
\hline \multirow[t]{2}{*}{ General index } & 626.1 & 648.1 & 676.9 & 131.1 & 136.2 \\
\hline & \multicolumn{5}{|c|}{ (Average percentage change) } \\
\hline Foodstuffs, beverages, and tobacco & 4.3 & 6.6 & 7.4 & 8.2 & 4.2 \\
\hline Clothing and shoes & -0.3 & -0.4 & -0.6 & 0.4 & 2.7 \\
\hline Housing costs & 3.2 & 1.0 & 1.2 & 2.7 & 1.9 \\
\hline Furniture & -0.3 & 0.2 & 0.2 & 1.8 & 3.5 \\
\hline Health and medical care & 1.0 & 3.0 & 2.2 & 3.4 & 2.8 \\
\hline Transportation and communications & 1.5 & -0.5 & 0.8 & 3.6 & 2.5 \\
\hline Education and entertainment & 0.1 & 1.8 & 3.8 & 6.0 & -0.1 \\
\hline Other services & 0.9 & 1.7 & 3.5 & 7.9 & 11.0 \\
\hline General index & 2.5 & 3.5 & 4.5 & 5.7 & 3.9 \\
\hline
\end{tabular}

Source: Algerian authorities.

$1 /$ Includes 256 items and covers households Algiers area.

2/ New Index base 2009 
Table 15. Algeria: Income of Households, 2006-10

(In billions of dinars)

\begin{tabular}{lrrrrr}
\hline & 2006 & 2007 & 2008 & 2009 & 2010 \\
\hline Wages and salaries 1/ & $1,411.7$ & $1,698.7$ & $2,108.1$ & $2,284.7$ & $\ldots$ \\
$\quad$ Agriculture & 75.3 & 89.5 & 95.3 & 111.9 & $\ldots$ \\
Central government & 681.5 & 797.2 & $1,127.5$ & $1,156.2$ & $\ldots$ \\
$\quad$ Other sectors & 654.9 & 812.0 & 885.3 & $1,016.6$ & $\ldots$ \\
Self-employed & $1,900.7$ & $2,176.0$ & $2,465.4$ & $2,935.4$ & $\ldots$ \\
Transfers & 828.0 & $1,309.5$ & $1,148.6$ & $1,356.7$ & $\ldots$ \\
Gross income & $4,140.4$ & $5,184.2$ & $5,722.1$ & $1,356.7$ & $\ldots$ \\
Disposable income & $3,587.1$ & $4,539.5$ & $4,908.4$ & $6,576.8$ & $\ldots$ \\
\hline
\end{tabular}

Source: Algerian authorities.

$1 /$ Includes social security contributions paid by employees. 
Table 16. Algeria: Labor Force, Employment, and Unemployment, 2006-10 1/

(In thousands; unless otherwise indicated)

\begin{tabular}{lrrrrr}
\hline & 2006 & 2007 & 2008 & 2009 & 2010 \\
\hline Labor force & 10,267 & 10,514 & 10,801 & 10,544 & 10,812 \\
Agriculture & 1,780 & 1,842 & 1,841 & 1,242 & 1,136 \\
Other sectors & 8,487 & 8,672 & 8,960 & 9,302 & 9,676 \\
Employment & 6,517 & 6,771 & 7,002 & 9,472 & 9,736 \\
Agriculture & 1,780 & 1,842 & 1,841 & 1,242 & 1,136 \\
Other sectors & 4,737 & 4,929 & 5,161 & 8,230 & 8,600 \\
$\quad$ Industry & 525 & 522 & 530 & 1,194 & 1,337 \\
$\quad$ Construction and public works & 1,160 & 1,261 & 1,371 & 1,718 & 1,886 \\
$\quad$ Government & 1,542 & 1,557 & 1,572 & 5,318 & 5,377 \\
$\quad$ Other & 1,510 & 1,589 & 1,688 & $\ldots$ & $\ldots$ \\
Work at home 2/ & 2,485 & 2,498 & 2,579 & $\ldots$ & $\ldots$ \\
Unemployed & 1,265 & 1,245 & 1,220 & 1,072 & 1,076 \\
$\quad$ in percent of labor force) & 12.3 & 12 & 11 & 10 & 10 \\
Youth unemployment 3/ & 24.3 & 25 & 24 & 21 & 21.5 \\
Urban youth unemployment & 28.6 & 27 & 25 & $\ldots$ & $\ldots$ \\
\hline
\end{tabular}

Source: Algerian authorities; and Fund staff estimates.

1/ Data not strictly comparable over time.

2/ Including military draft and irregular employment. The increase in 2004 possibly reflects in part the occurrence of Ramadan shortly after the annual household survey was conducted.

$3 /$ Unemployed of less than 24 years of age in percent of labor force of less than 24 years of age. 
Table 17. Algeria: Summary of Central Government Operations, 2006-10

(In billions of dinars)

\begin{tabular}{|c|c|c|c|c|c|}
\hline & 2006 & 2007 & 2008 & 2009 & 2010 \\
\hline Total budget revenue and grants $1 /$ & $3,639.9$ & $3,687.9$ & $5,190.6$ & $3,675.3$ & $4,382.5$ \\
\hline Hydrocarbon revenue 2/ & $2,799.0$ & $2,796.8$ & $4,088.6$ & $2,412.7$ & $2,905.0$ \\
\hline Nonhydrocarbon revenue $1 /$ & 840.8 & 890.9 & $1,101.9$ & $1,262.5$ & $1,477.4$ \\
\hline Tax revenue & 720.9 & 766.8 & 965.3 & $1,146.6$ & $1,287.5$ \\
\hline Taxes on income and profits & 241.2 & 258.1 & 331.5 & 462.2 & 561.7 \\
\hline Taxes on goods and services & 341.3 & 347.4 & 435.2 & 478.4 & 504.3 \\
\hline Customs duties & 114.8 & 133.1 & 164.9 & 170.2 & 181.9 \\
\hline Registration and stamps & 23.5 & 28.1 & 33.6 & 35.8 & 39.7 \\
\hline Nontax revenues $1 /$ & 119.9 & 124.1 & 136.7 & 115.9 & 189.9 \\
\hline Fees 1/ & 44.3 & 75.2 & 113.8 & 66.8 & 64.4 \\
\hline Bank of Algeria dividends & 75.3 & 41.0 & 22.5 & 48.2 & 121.1 \\
\hline Dividends from holdings & 0.3 & 7.9 & 0.3 & 0.9 & 4.4 \\
\hline Grants & 0.1 & 0.2 & 0.1 & 0.1 & 0.1 \\
\hline Total budget expenditure & $2,454.4$ & $3,114.2$ & $4,191.2$ & $4,185.3$ & $4,502.4$ \\
\hline Current expenditure & $1,435.2$ & $1,672.0$ & $2,218.0$ & $2,259.5$ & $2,694.5$ \\
\hline Personnel expenditure & 531.3 & 628.7 & 826.6 & 879.9 & $1,193.1$ \\
\hline Mudjahidins' pensions & 92.5 & 101.6 & 103.0 & 130.7 & 151.3 \\
\hline Material and supplies & 95.7 & 93.8 & 111.7 & 112.5 & 121.8 \\
\hline Current transfers $3 /$ & 645.6 & 762.8 & $1,115.2$ & $1,098.9$ & $1,195.8$ \\
\hline Interest payments & 70.0 & 85.0 & 61.4 & 37.4 & 32.5 \\
\hline Capital expenditure & $1,019.2$ & $1,442.3$ & $1,973.3$ & $1,925.8$ & $1,807.9$ \\
\hline Budget balance & $1,185.5$ & 573.6 & 999.4 & -510.0 & -119.9 \\
\hline Special accounts balance & -4.1 & -18.9 & -31.2 & 4.3 & -34.7 \\
\hline Net lending by Treasury & 32.1 & 141.31 & 123.8 & 138.5 & 138.9 \\
\hline Primary balance 4/ & $1,219.3$ & 498.4 & 905.8 & -606.8 & -260.9 \\
\hline Overall balance 4/ & $1,149.3$ & 413.4 & 844.4 & -644.2 & -293.5 \\
\hline Financing & $-1,149.3$ & -413.4 & -844.4 & 644.2 & 293.5 \\
\hline Domestic 5/ & -990.7 & -302.8 & -821.7 & 665.8 & 308.3 \\
\hline Foreign 6/ & -158.6 & -110.7 & -22.6 & -21.6 & -14.9 \\
\hline
\end{tabular}

Sources: Algerian authorities; and Fund staff estimates.

1/ Excluding privatization receipts, which are classified under nonbank financing.

2/ Including dividends on current profits paid by Sonatrach.

3/ Covers expenditures for public services, food subsidies, agricultural price support, and cash transfers forthe poor.

4/ Including special accounts, net lending and operations of the Rehabilitation Fund.

$5 /$ Including debt rescheduling proceeds blocked on account at Bank of Algeria.

$6 /$ Includes external debt rescheduling proceeds. 
Table 18. Algeria: Composition of Central Government Revenue, 2006-10

\begin{tabular}{|c|c|c|c|c|c|}
\hline & 2006 & 2007 & 2008 & 2009 & 2010 \\
\hline & \multicolumn{5}{|c|}{ (In billions of dinars) } \\
\hline Total budget revenue and grants $1 /$ & $3,639.9$ & $3,687.9$ & $5,190.6$ & $3,675.3$ & $4,382.5$ \\
\hline Hydrocarbon revenue & $2,799.0$ & $2,796.8$ & $4,088.6$ & $2,412.7$ & $2,905.0$ \\
\hline Of which: Sonatrach dividends & 85.0 & 85.0 & 85.0 & 85.0 & 85.0 \\
\hline Nonhydrocarbon revenue $1 /$ & 840.8 & 890.9 & $1,101.9$ & $1,262.5$ & $1,477.4$ \\
\hline Tax revenue & 720.9 & 766.8 & 965.3 & $1,146.6$ & $1,287.5$ \\
\hline Taxes on income and profits & 241.2 & 258.1 & 331.5 & 462.2 & 561.7 \\
\hline Wage income taxes & 96.1 & 124.9 & 155.5 & 183.6 & 244.8 \\
\hline Other & 145.1 & 133.2 & 176.0 & 278.6 & 316.9 \\
\hline Taxes on goods and services & 341.3 & 347.4 & 435.2 & 478.4 & 504.3 \\
\hline VAT and excises on imports & 140.9 & 171.9 & 223.3 & 234.5 & 252.6 \\
\hline VAT and excises on domestic activities & 145.7 & 168.8 & 196.8 & 233.6 & 232.9 \\
\hline VAT on domestic transactions & 114.2 & 136.6 & 165.3 & 199.7 & 199.7 \\
\hline Tobacco and alcohol excises (DIC) & 31.5 & 32.2 & 31.5 & 33.9 & 33.2 \\
\hline VAT on petroleum products/levy & 4.4 & 0.1 & 0.1 & 0.0 & 0.2 \\
\hline Excises on petroleum products & 40.6 & 4.1 & 0.2 & 0.7 & 0.7 \\
\hline Other indirect taxes & 9.615 & 2.5 & 14.8 & 10.4 & 28.7 \\
\hline Customs duties & 114.8 & 133.1 & 164.9 & 169.1 & 169.1 \\
\hline Registration and stamps & 23.5 & 28.1 & 33.6 & 35.8 & 39.7 \\
\hline Nontax revenue $1 /$ & 119.9 & 124.1 & 136.7 & 115.9 & 189.9 \\
\hline Fees 1/ & 44.3 & 75.2 & 113.8 & 66.8 & 64.4 \\
\hline Bank of Algeria dividends & 75.3 & 41.0 & 22.5 & 48.2 & 121.1 \\
\hline Dividends from holdings & 0.3 & 7.9 & 0.3 & 0.9 & 4.4 \\
\hline \multirow[t]{2}{*}{ Grants } & 0.1 & 0.2 & 0.1 & 0.1 & 0.1 \\
\hline & \multicolumn{5}{|c|}{ (In percent of total budget revenue and grants) } \\
\hline Total budget revenue and grants & 100.0 & 100.0 & 100.0 & 100.0 & 100.0 \\
\hline Hydrocarbon revenue & 76.9 & 75.8 & 78.8 & 65.6 & 66.3 \\
\hline Nonhydrocarbon revenue & 23.1 & 24.2 & 21.2 & 34.4 & 33.7 \\
\hline Tax revenue & 19.8 & 20.8 & 18.6 & 31.2 & 29.4 \\
\hline Taxes on income and profits & 6.6 & 7.0 & 6.4 & 12.6 & 12.8 \\
\hline Taxes on goods and services & 9.4 & 9.4 & 8.4 & 13.0 & 11.5 \\
\hline Customs duties & 3.2 & 3.6 & 3.2 & 4.6 & 3.9 \\
\hline Registration and stamps & 0.6 & 0.8 & 0.6 & 1.0 & 0.9 \\
\hline Nontax revenue & 3.3 & 3.4 & 2.6 & 3.2 & 4.3 \\
\hline Grants & 0.0 & 0.0 & 0.0 & 0.0 & 0.0 \\
\hline
\end{tabular}

Sources: Algerian authorities; and Fund staff estimates.

1/ Excluding privatization receipts. 
Table 19. Algeria: Central Government Revenue, 2006-10

(In percent of GDP)

\begin{tabular}{lrrrrr}
\hline & 2006 & 2007 & 2008 & 2009 & 2010 \\
\hline Total budget revenue and grants 1/ & 42.7 & 39.6 & 47.2 & 36.6 & 36.4 \\
Hydrocarbon revenue & 32.8 & 30.1 & 37.2 & 24.0 & 24.1 \\
Of which: Sonatrach dividends & 1.0 & 0.9 & 0.8 & 0.8 & 0.7 \\
Nonhydrocarbon revenue 1/ & 9.9 & 9.6 & 10.0 & 12.6 & 12.3 \\
Tax revenue & 8.5 & 8.2 & 8.8 & 11.4 & 10.7 \\
Taxes on income and profits & 2.8 & 2.8 & 3.0 & 4.6 & 4.7 \\
Wage income taxes & 1.1 & 1.3 & 1.4 & 1.8 & 2.0 \\
Other & 1.7 & 1.4 & 1.6 & 2.8 & 2.6 \\
Taxes on goods and services & 4.0 & 3.7 & 4.0 & 4.8 & 4.2 \\
VAT and excises on imports & 1.7 & 1.8 & 2.0 & 2.3 & 2.1 \\
VAT and excises on domestic activitie & 1.7 & 1.8 & 1.8 & 2.3 & 1.9 \\
VAT on domestic transactions & 1.3 & 1.5 & 1.5 & 2.0 & 1.7 \\
Tobacco and alcohol excises (DIC) & 0.4 & 0.3 & 0.3 & 0.3 & 0.3 \\
VAT on petroleum products/lew & 0.1 & 0.0 & 0.0 & 0.0 & 0.0 \\
Excises on petroleum products & 0.5 & 0.0 & 0.0 & 0.0 & 0.0 \\
Other indirect taxes & 0.1 & 0.0 & 0.1 & 0.1 & 0.2 \\
Customs duties & 1.3 & 1.4 & 1.5 & 1.7 & 1.5 \\
Registration and stamps & 0.3 & 0.3 & 0.3 & 0.4 & 0.3 \\
Nontax revenue 1/ & 1.4 & 1.3 & 1.2 & 1.2 & 1.6 \\
Fees 1/ & 0.5 & 0.8 & 1.0 & 0.7 & 0.5 \\
Bank of Algeria dividends & 0.9 & 0.4 & 0.2 & 0.5 & 1.0 \\
Dividends from holdings & 0.0 & 0.1 & 0.0 & 0.0 & 0.0 \\
\hline
\end{tabular}

Source: Algerian authorities.

1/ Excluding privatization receipts. 
Table 20. Algeria: Composition of Central Government Expenditure, 2006-10

\begin{tabular}{|c|c|c|c|c|c|}
\hline & 2006 & 2007 & 2008 & 2009 & 2010 \\
\hline & \multicolumn{5}{|c|}{ (In billions of dinars) } \\
\hline Budget expenditure & $2,454.4$ & $3,114.2$ & $4,191.2$ & $4,185.3$ & $4,502.4$ \\
\hline Current expenditure & $1,435.2$ & $1,672.0$ & $2,218.0$ & $2,259.5$ & $2,694.5$ \\
\hline Personnel expenditure & 531.3 & 628.7 & 826.6 & 879.9 & $1,193.1$ \\
\hline Wages and salaries & 531.3 & 628.7 & 826.6 & 879.9 & $1,193.1$ \\
\hline Other & 0.0 & 1.0 & 2.0 & 0 & 0 \\
\hline Mudjahidins' pensions & 92.5 & 101.6 & 103.0 & 130.7 & 151.3 \\
\hline Material and supplies & 95.7 & 93.8 & 111.7 & 112.5 & 121.8 \\
\hline Public services & 215.5 & 273.0 & 360.8 & 412.54 & 513.2 \\
\hline Hospitals & 73.5 & 107.9 & 151.5 & 176.0 & 197.2 \\
\hline Other & 142 & 165.2 & 209.4 & 236.5 & 316 \\
\hline Current transfers & 430.1 & 489.8 & 824.7 & 839.0 & 795.5 \\
\hline Public works and social assistanc€ & 0.0 & 0.0 & 0.0 & 0.0 & 0.0 \\
\hline Food subsidies & 1.1 & 1.2 & 2.5 & 2.5 & 2.6 \\
\hline Youth Employment Support Fund & 5.5 & 5.5 & 5.5 & 16.6 & 13.8 \\
\hline Other transfers & 423.5 & 483.1 & 816.7 & 819.9 & 779.1 \\
\hline Interest payments & 70.0 & 85.0 & 61.4 & 37.4 & 32.5 \\
\hline Capital expenditure & $1,019.2$ & $1,442.3$ & $1,973.3$ & $1,925.8$ & $1,807.9$ \\
\hline Special accounts balance & -4.1 & -18.9 & -31.2 & 4.3 & -34.7 \\
\hline Net lending by Treasury & 32.1 & 141.3 & 123.8 & 138.5 & 138.9 \\
\hline \multirow[t]{2}{*}{ Total expenditure and net lending } & $2,490.6$ & $3,274.4$ & $4,346.2$ & $4,319.5$ & $4,675.9$ \\
\hline & \multicolumn{5}{|c|}{ (In percent of total expenditure) } \\
\hline Total expenditure and net lending & 100.0 & 100.0 & 100.0 & 100.0 & 100.0 \\
\hline Budget expenditure & 98.5 & 95.1 & 96.4 & 96.9 & 96.3 \\
\hline Current expenditure & 57.6 & 51.1 & 51.0 & 52.3 & 57.6 \\
\hline Personnel expenditure & 21.3 & 19.2 & 19.0 & 20.4 & 25.5 \\
\hline Mudjahidins' pensions & 3.7 & 3.1 & 2.4 & 3.0 & 3.2 \\
\hline Material and supplies & 3.8 & 2.9 & 2.6 & 2.6 & 2.6 \\
\hline Public services & 8.7 & 8.3 & 8.3 & 9.6 & 11.0 \\
\hline Current transfers & 17.3 & 15.0 & 19.0 & 19.4 & 17.0 \\
\hline Interest payments & 2.8 & 2.6 & 1.4 & 0.9 & 0.7 \\
\hline Capital expenditure & 40.9 & 44.0 & 45.4 & 44.6 & 38.7 \\
\hline Special accounts balance & -0.2 & -0.6 & -0.7 & 0.1 & -0.7 \\
\hline Net lending by Treasury & 1.3 & 4.3 & 2.8 & 3.2 & 3.0 \\
\hline
\end{tabular}

Sources: Algerian authorities; and Fund staff estimates. 
Table 21. Algeria: Central Government Expenditure, 2006-10

(In percent of GDP)

\begin{tabular}{lrrrr}
\hline & 2007 & 2008 & 2009 & 2010 \\
\hline Total expenditure and net lending & 35.2 & 39.5 & 43.0 & 38.8 \\
Current expenditure & 18.0 & 20.2 & 22.5 & 22.4 \\
Personnel & 6.8 & 7.5 & 8.8 & 9.9 \\
Wages and salaries & 6.8 & 7.5 & 8.8 & 9.9 \\
Other & 0.0 & 0.0 & 0.0 & 0.0 \\
Mudjahidins' pensions & 1.1 & 0.9 & 1.3 & 1.3 \\
Material and supplies & 1.0 & 1.0 & 1.1 & 1.0 \\
Public services & 2.9 & 3.3 & 4.1 & 4.3 \\
$\quad$ Hospitals & 1.2 & 1.4 & 1.8 & 1.6 \\
Other & 1.8 & 1.9 & 2.4 & 2.6 \\
Current transfers & 5.3 & 6.9 & 6.8 & 5.7 \\
$\quad$ Public works and social assistance & 0.0 & 0.0 & 0.0 & 0.0 \\
Food subsidies & 0.0 & 0.0 & 0.0 & 0.0 \\
Youth Employment Support Fund & 0.1 & 0.5 & 0.3 & 0.3 \\
Other transfers & 5.3 & 5.9 & 5.9 & 4.9 \\
Interest on debt & 0.9 & 0.6 & 0.4 & 0.3 \\
Capital expenditure & 15.5 & 17.9 & 19.2 & 15.0 \\
Special accounts balance & -0.2 & -0.3 & 0.0 & -0.3 \\
Net lending by Treasury & 1.5 & 1.1 & 1.4 & 1.2 \\
\hline
\end{tabular}

Sources: Algerian authorities; and Fund staff estimates. 
Table 22. Algeria: Sectoral Allocation of Budgetary Capital Expenditure, 2006-10 1/

\begin{tabular}{|c|c|c|c|c|c|}
\hline & 2006 & 2007 & 2008 & 2009 & 2010 \\
\hline & \multicolumn{5}{|c|}{ (In millions of dinars) } \\
\hline Agriculture and fishery & 12,463 & 15,099 & 26,390 & 25,769 & 22,388 \\
\hline Irrigation and waterworks & 169,108 & 183,897 & 221,022 & 256,554 & 272,113 \\
\hline Industry and energy & 130 & 200 & 24 & 134 & 164 \\
\hline Tourism & 24 & 17 & 100 & 374 & 506 \\
\hline Economic infrastructure & 225,456 & 380,386 & 539,244 & 398,510 & 381,673 \\
\hline Housing & 89,683 & 184,746 & 187,570 & 230,758 & 293,495 \\
\hline Education and professional training & 84,136 & 126,160 & 135,710 & 144,913 & 153,513 \\
\hline Social infrastructure & 32,900 & 41,760 & 54,885 & 68,483 & 71,235 \\
\hline Administrative infrastructure & 38,446 & 54,439 & 80,365 & 85,665 & 113,726 \\
\hline Urban development & 51,056 & 79,350 & 78,911 & 77,613 & 65,349 \\
\hline Unallocated & 91,060 & 111,622 & 116,597 & 136,736 & 134,446 \\
\hline \multirow[t]{2}{*}{ Total } & 794,462 & $1,177,676$ & $1,440,818$ & $1,425,509$ & $1,508,608$ \\
\hline & \multicolumn{5}{|c|}{ (Annual percentage change) } \\
\hline Agriculture and fishery & -4.9 & 21.2 & 74.8 & -2.4 & -13.1 \\
\hline Irrigation and waterworks & 34.8 & 8.7 & 20.2 & 16.1 & 6.1 \\
\hline Industry and energy & 36.8 & 53.8 & -88.0 & 458.3 & 22.4 \\
\hline Tourism & 300.0 & -29.2 & 488.2 & 274.0 & 35.3 \\
\hline Economic infrastructure & 45.2 & 68.7 & 41.8 & -26.1 & -4.2 \\
\hline Housing & 32.8 & 106.0 & 1.5 & 23.0 & 27.2 \\
\hline Education and professional training & -0.5 & 49.9 & 7.6 & 6.8 & 5.9 \\
\hline Social infrastructure & -7.5 & 26.9 & 31.4 & 24.8 & 4.0 \\
\hline Administrative infrastructure & 28.3 & 41.6 & 47.6 & 6.6 & 32.8 \\
\hline Urban development & 37.1 & 55.4 & -0.6 & -1.6 & -15.8 \\
\hline Unallocated & 15.5 & 22.6 & 4.5 & 17.3 & -1.7 \\
\hline \multirow[t]{2}{*}{ Total } & 26.6 & 48.2 & 22.3 & -1.1 & 5.8 \\
\hline & \multicolumn{5}{|c|}{ (In percent of total) } \\
\hline Agriculture and fishery & 1.6 & 1.3 & 1.8 & 1.8 & 1.5 \\
\hline Irrigation and waterworks & 21.3 & 15.6 & 15.3 & 18.0 & 18.0 \\
\hline Industry and energy & 0.0 & 0.0 & 0.0 & 0.0 & 0.0 \\
\hline Tourism & 0.0 & 0.0 & 0.0 & 0.0 & 0.0 \\
\hline Economic infrastructure & 28.4 & 32.3 & 37.4 & 28.0 & 25.3 \\
\hline Housing & 11.3 & 15.7 & 13.0 & 16.2 & 19.5 \\
\hline Education and professional training & 10.6 & 10.7 & 9.4 & 10.2 & 10.2 \\
\hline Social infrastructure & 4.1 & 3.5 & 3.8 & 4.8 & 4.7 \\
\hline Administrative infrastructure & 4.8 & 4.6 & 5.6 & 6.0 & 7.5 \\
\hline Urban development & 6.4 & 6.7 & 5.5 & 5.4 & 4.3 \\
\hline Unallocated & 11.5 & 9.5 & 8.1 & 9.6 & 8.9 \\
\hline Total & 100.0 & 100.0 & 100.0 & 100.0 & 100.0 \\
\hline
\end{tabular}

Source: Algerian authorities.

1/ Commitment basis. 
Table 23. Algeria: Central Government Domestic Debt, 2006-10

(In billions of dinars; end-of-period)

\begin{tabular}{lrrrrr}
\hline & 2006 & 2007 & 2008 & 2009 & 2010 \\
\hline Total domestic debt 1/ & $1,847.3$ & $1,103.9$ & 734.0 & 816.3 & 1107.4 \\
Equipment bonds and regular securities & $1,066.9$ & $1,052.7$ & 677.8 & 760.1 & $1,051.2$ \\
Central bank overdrafts & 729.2 & 0.0 & 0.0 & 0.0 & 0.0 \\
Refinancing bonds & 51.2 & 51.2 & 56.2 & 56.2 & 56.2 \\
Public enterprises, EPIC, agroindustries & 0.0 & 0.0 & 0.0 & 0.0 & 0.0 \\
Supplementary restructuring debt & 0.0 & 0.0 & 0.0 & 0.0 & 0.0 \\
OPGI (real estate companies) & 0.0 & 0.0 & 0.0 & 0.0 & 0.0 \\
Farmers' debt & 0.0 & 0.0 & 0.0 & 0.0 & 0.0 \\
Banks & 51.2 & 51.2 & 56.2 & 56.2 & 56.2 \\
Solidarity bonds & 0.0 & 0.0 & 0.0 & 0.0 & 0.0 \\
CNAC (unemployment insurance agency) & 0.0 & 0.0 & 0.0 & 0.0 & 0.0 \\
Bonds (CNR-retirement insurance agency) & 0.0 & 0.0 & 0.0 & 0.0 & 0.0 \\
Bonds (CNAS-social security agency) & 0.0 & 0.0 & 0.0 & 0.0 & 0.0 \\
Memorandum items: & & & & & \\
Domestic debt as percent of GDP & 21.7 & 11.8 & 6.6 & 8.1 & 9.2 \\
GDP (In billions of dinars) & 8,515 & 9,367 & 11,090 & 10,034 & 12,050 \\
\hline
\end{tabular}

Source: Algerian authorities.

1/ Excluding the blocked account at the Bank of Algeria and other deposits. 
Table 24. Algeria: Monetary Survey, 2006-10 1/

\begin{tabular}{|c|c|c|c|c|c|}
\hline & 2006 & 2007 & 2008 & 2009 & 2010 \\
\hline & \multicolumn{5}{|c|}{ (In billions of dinars) } \\
\hline Foreign assets (net) & $5,515.0$ & $7,415.5$ & $10,246.9$ & $10,886.0$ & $11,996.9$ \\
\hline Central bank & $5,526.3$ & $7,382.9$ & $10,227.5$ & $10,865.9$ & $12,005.6$ \\
\hline Commercial banks & -11.3 & 32.6 & 19.4 & 20.1 & -8.7 \\
\hline Net domestic assets & -581.4 & $-1,420.9$ & $-3,291.0$ & $-3,712.9$ & $-3,834.1$ \\
\hline Domestic credit & 601.3 & 12.1 & $-1,011.8$ & -402.4 & -242.8 \\
\hline Credit to government (net) & $-1,304.1$ & $-2,193.1$ & $-3,627.3$ & $-3,488.9$ & $-3,510.9$ \\
\hline Central bank 2/ & $-2,510.7$ & $-3,294.9$ & $-4,365.7$ & $-4,402.0$ & $-4,930.2$ \\
\hline Commercial banks $3 /$ & 870.8 & 723.1 & 278.6 & 340.2 & 683.8 \\
\hline CCP and treasury deposits & 335.8 & 378.7 & 459.8 & 572.9 & 735.5 \\
\hline Credit to the economy $3 /$ & $1,905.4$ & $2,205.2$ & $2,615.5$ & $3,086.5$ & $3,268.1$ \\
\hline Government lending funds & -33.5 & -28.9 & -16.3 & -14.8 & -14.5 \\
\hline Medium- and long-term foreign liabilities & -19.4 & -14.7 & -11.4 & -8.5 & -6.6 \\
\hline Other items (net) & $-1,129.8$ & $-1,389.4$ & $-2,251.5$ & $-3,287.2$ & $-3,570.2$ \\
\hline Money plus quasi-money (M2) & $4,933.7$ & $5,994.6$ & $6,955.9$ & $7,173.1$ & $8,162.8$ \\
\hline Money & $3,167.6$ & $4,233.6$ & $4,964.9$ & $4,944.2$ & $5,638.5$ \\
\hline Currency outside banks & $1,081.4$ & $1,284.5$ & $1,540.0$ & $1,829.4$ & $2,098.6$ \\
\hline Demand deposits & $1,750.4$ & $2,570.4$ & $2,965.1$ & $2,541.9$ & $2,804.4$ \\
\hline \multicolumn{6}{|l|}{ Deposits with treasury and postal } \\
\hline \multirow[t]{2}{*}{ Quasi-money } & $1,766.1$ & $1,761.0$ & $1,991.0$ & $2,228.9$ & $2,524.3$ \\
\hline & \multicolumn{5}{|c|}{ (Annual percentage change) } \\
\hline Money and quasi-money & 19.1 & 21.5 & 16.0 & 3.1 & 13.8 \\
\hline Money & 31.0 & 33.7 & 17.3 & -0.4 & 14.0 \\
\hline Quasi-money & 2.4 & -0.3 & 13.1 & 11.9 & 13.3 \\
\hline Net domestic assets & $1,441.5$ & 144.4 & 131.6 & 12.8 & 3.3 \\
\hline Domestic credit & -27.9 & -98.0 & $-8,462.0$ & -60.2 & -39.7 \\
\hline Credit to government 3/ & 38.1 & 68.2 & 65.4 & -3.8 & 0.6 \\
\hline \multirow[t]{2}{*}{ Credit to the economy $3 /$} & 7.1 & 15.7 & 18.6 & 18.0 & 5.9 \\
\hline & \multicolumn{5}{|c|}{ (In percent) } \\
\hline \multicolumn{6}{|l|}{ Memorandum items: } \\
\hline Total liquidity/GDP & 57.9 & 64.0 & 62.7 & 71.5 & 67.7 \\
\hline Money/GDP & 37.2 & 45.2 & 44.8 & 49.3 & 46.8 \\
\hline Currency outside banks/GDP & 12.7 & 13.7 & 13.9 & 18.2 & 17.4 \\
\hline Domestic credit/GDP & 7.1 & 0.1 & -9.1 & -4.0 & -2.0 \\
\hline Money/M2 & 64.2 & 70.6 & 71.4 & 68.9 & 69.1 \\
\hline Currency outside banks/M2 & 21.9 & 21.4 & 22.1 & 25.5 & 25.7 \\
\hline Money multiplier & 3.7 & 3.5 & 3.6 & 3.2 & 3.1 \\
\hline Nominal GDP (in billions of dinars) & $8,514.8$ & $9,366.5$ & $11,090.0$ & $10,034.3$ & $12,049.5$ \\
\hline
\end{tabular}

Sources: Bank of Algeria; and Fund staff estimates.

$1 /$ Including savings banks. Money and quasi-money include deposits of nonbank financial in stitutions in the banking system.

2/ Includes, as a net item, deposits of the Banque Algérienne de Développement and ministries with the central bank.

$3 /$ Includes impact of banks' restructuring packages. The conversion of banks' claims on public enterprises in banks' claims on the government results, other things being equal, in a decrease in credit to the economy and an equal increase in credit to the government. 
Table 25. Algeria: Summary Balance Sheet of the Bank of Algeria, 2006-10

\begin{tabular}{|c|c|c|c|c|c|}
\hline & 2006 & 2007 & 2008 & 2009 & 2010 \\
\hline & \multicolumn{5}{|c|}{ (In billions of dinars) } \\
\hline Net foreign assets & $5,526.3$ & $7,382.9$ & $10,227.5$ & $10,864.9$ & $12,005.6$ \\
\hline Net domestic assets & $-4,191.0$ & $-5,653.8$ & $-8,302.0$ & $-8,651.7$ & $-9,399.7$ \\
\hline Credit to government & $-2,510.7$ & $-3,294.8$ & $-4,365.7$ & $-4,402.0$ & $-4,930.2$ \\
\hline Credit to banks & 0.0 & 0.0 & 0.0 & 0.0 & 0.0 \\
\hline Other credit & 1.3 & 1.5 & 1.5 & 1.4 & 1.4 \\
\hline Other items net & $-1,681.6$ & $-2,360.5$ & $-3,937.8$ & $-4,251.1$ & $-4,470.9$ \\
\hline Reserve money & $1,335.3$ & $1,729.0$ & $1,925.5$ & $2,214.2$ & $2,606.0$ \\
\hline Currency in circulation & $1,092.1$ & $1,301.3$ & $1,561.7$ & $1,849.9$ & $2,132.2$ \\
\hline \multirow[t]{2}{*}{ Bankers deposits $1 /$} & 243.2 & 427.7 & 363.8 & 364.3 & 473.8 \\
\hline & \multicolumn{5}{|c|}{ (Annual change; in billions of dinars) } \\
\hline Net foreign assets & $1,374.8$ & $1,856.6$ & $2,844.6$ & 638.4 & $1,139.8$ \\
\hline Net domestic assets & $-1,202.8$ & $-1,462.8$ & $-2,648.2$ & -349.7 & -748.0 \\
\hline Credit to government & -524.2 & -784.1 & $-1,070.9$ & -36.3 & -528.2 \\
\hline Credit to banks & 0.0 & 0.0 & 0.0 & 0.0 & 0.0 \\
\hline Other credit & 0.5 & 0.2 & 0.0 & -0.1 & 0.0 \\
\hline Other items net & -679.1 & -678.9 & $-1,577.3$ & -313.3 & -219.8 \\
\hline Reserve money & 172.0 & 393.7 & 196.5 & 288.7 & 391.8 \\
\hline Currency in circulation & 161.8 & 209.2 & 260.4 & 288.2 & 282.3 \\
\hline \multirow[t]{2}{*}{ Bankers deposits $1 /$} & 10.1 & 184.5 & -63.9 & 0.5 & 109.5 \\
\hline & \multicolumn{5}{|c|}{ (Annual percentage change) } \\
\hline Net foreign assets & 33.1 & 33.6 & 38.5 & 6.2 & 10.5 \\
\hline Net domestic assets & 40.3 & 34.9 & 46.8 & 4.2 & 8.6 \\
\hline Credit to government & 26.4 & 31.2 & 32.5 & 0.8 & 12.0 \\
\hline Credit to banks & 1.0 & 2.0 & 3.0 & 3.0 & 4.0 \\
\hline Reserve money & 14.8 & 29.5 & 11.4 & 15.0 & 17.7 \\
\hline Currency in circulation & 17.4 & 19.2 & 20.0 & 18.5 & 15.3 \\
\hline Bankers deposits $1 /$ & 4.4 & 75.8 & -14.9 & 0.1 & 30.1 \\
\hline
\end{tabular}

Source: Bank of Algeria.

$1 /$ Includes deposits of nonbank financial institutions. 
Table 26. Algeria: Summary Balance Sheet of Deposit Money Banks, 2006-10

\begin{tabular}{|c|c|c|c|c|c|c|}
\hline & 2005 & 2006 & 2007 & 2008 & 2009 & 2010 \\
\hline & \multicolumn{6}{|c|}{ (In billions of dinars) } \\
\hline Net foreign assets & 27.9 & -11.3 & 3.5 & 16.8 & 11.6 & -15.2 \\
\hline Assets & 91.6 & 83.9 & 44.6 & 71.1 & 58.0 & 28.7 \\
\hline Liabilities & 63.6 & 95.3 & 41.2 & 54.3 & 46.4 & 43.9 \\
\hline Net domestic assets & $2,916.6$ & $3,530.8$ & $4,232.9$ & $5,011.0$ & $4,938.8$ & $5,565.6$ \\
\hline Domestic credit & $2,543.3$ & $2,774.9$ & $2,928.3$ & $2,894.1$ & $3,426.7$ & $3,951.9$ \\
\hline Credit to government & 765.9 & 870.8 & 723.1 & 278.6 & 340.2 & 683.8 \\
\hline Credit to the economy & $1,777.4$ & $1,904.1$ & $2,205.2$ & $2,615.5$ & $3,086.5$ & $3,268.1$ \\
\hline Of which: private sector & 881.6 & $1,055.7$ & $1,216.0$ & $1,413.3$ & $1,600.6$ & $1,806.7$ \\
\hline Claims on central bank & 684.8 & $1,169.9$ & $1,969.5$ & $2,848.9$ & $2,441.7$ & $2,528.8$ \\
\hline Central bank deposit auction & 499.8 & 907.0 & $1,583.1$ & $2,500.4$ & $2,122.1$ & $2,097.7$ \\
\hline Other deposits & 185.1 & 262.9 & 386.4 & 348.5 & 319.6 & 431.1 \\
\hline Of which: required reserves & 171.5 & 209.0 & 272.1 & 224.9 & 239.7 & 323.4 \\
\hline Cash in vault & 9.2 & 10.7 & 16.3 & 21.7 & 20.6 & 33.6 \\
\hline Other items (net) & -368.0 & -424.7 & -681.2 & -753.7 & -950.2 & -948.7 \\
\hline Of which: MLT foreign liabilities & -20.6 & -19.4 & -14.7 & -11.4 & -8.5 & -6.6 \\
\hline \multicolumn{7}{|l|}{ Domestic liabilities } \\
\hline Demand deposits & $1,220.4$ & $1,750.4$ & $2,570.4$ & $2,965.1$ & $2,541.9$ & $2,804.4$ \\
\hline Time deposits & $1,724.2$ & $1,769.1$ & $1,761.0$ & $1,991.0$ & $2,228.9$ & $2,524.3$ \\
\hline \multirow[t]{2}{*}{ Central bank refinancing } & 0.0 & 0.0 & 0.0 & 0.0 & 0.0 & 0.0 \\
\hline & \multicolumn{6}{|c|}{ (Annual change; in billions of dinars) } \\
\hline Net foreign assets & 17.8 & -39.2 & 14.8 & 13.4 & -5.2 & -26.8 \\
\hline Net domestic assets & 221.3 & 614.2 & 702.1 & 778.1 & -72.2 & 626.8 \\
\hline Credit to government & 29.0 & 104.9 & -147.7 & -444.5 & 61.6 & 343.6 \\
\hline Credit to the economy & 243.1 & 126.7 & 301.1 & 410.3 & 471.0 & 181.6 \\
\hline Claims on central bank & 12.4 & 485.0 & 799.6 & 879.4 & -407.2 & 87.1 \\
\hline Other & -109.7 & -55.2 & -250.9 & -67.1 & -197.6 & 14.5 \\
\hline \multirow[t]{2}{*}{ Domestic liabilities } & 239.2 & 575.0 & 811.9 & 624.7 & -185.3 & 557.9 \\
\hline & \multicolumn{6}{|c|}{ (Annual percentage change) } \\
\hline Foreign assets & 19.5 & -8.3 & -46.8 & 59.4 & -18.4 & -50.5 \\
\hline Foreign liabilities & -4.3 & 49.7 & -56.8 & 31.8 & -14.5 & -5.4 \\
\hline Net domestic assets & 8.2 & 21.1 & 19.9 & 18.4 & -1.4 & 12.7 \\
\hline Credit to government & 3.9 & 13.7 & -17.0 & -61.5 & 22.1 & 101.0 \\
\hline Credit to the economy & 15.8 & 7.1 & 15.8 & 18.6 & 18.0 & 5.9 \\
\hline Claims on central bank & 1.8 & 70.8 & 68.4 & 44.7 & -14.3 & 3.6 \\
\hline Other & 44.1 & 15.4 & 60.6 & 10.1 & 27.0 & -1.6 \\
\hline Domestic liabilities & 8.8 & 19.5 & 23.1 & 14.4 & -3.7 & 11.7 \\
\hline
\end{tabular}

Source: Bank of Algeria. 
Table 27. Algeria: Distribution of Credit to the Economy by Maturity, 2006-10 1/

\begin{tabular}{lrrrrr}
\hline & 2006 & 2007 & 2008 & 2009 & 2010 \\
\hline & \multicolumn{5}{c}{ (In billions of dinars; end-of-period) } \\
Short-term & 915.7 & $1,026.1$ & $1,189.4$ & $1,320.5$ & $1,311.0$ \\
Medium-term & 786.4 & 828.0 & 910.0 & 852.1 & 904 \\
Long-term & 203.3 & 351.1 & 516.1 & 913.90 & $1,053.1$ \\
Total & $1,905.4$ & $2,205.2$ & $2,615.5$ & $3,086.5$ & $3,268.1$ \\
& \multicolumn{5}{c}{ (Annual percentage change) } \\
Short-term & -0.8 & 12.1 & 15.9 & 11.0 & -0.7 \\
Medium-term & 5.2 & 5.3 & 9.9 & -6.4 & 6.1 \\
Long-term & 86.5 & 72.7 & 47.0 & 77.1 & 15.2 \\
Total & 7.1 & 15.7 & 18.6 & 18.0 & 5.9 \\
& \multicolumn{7}{c}{ (In percent of total credit) } \\
Short-term & 48.1 & 46.5 & 45.5 & 42.8 & 40.1 \\
Medium-term & 41.3 & 37.5 & 34.8 & 27.6 & 27.7 \\
Long-term & 10.7 & 15.9 & 19.7 & 29.6 & 32.2 \\
Total & 100.0 & 100.0 & 100.0 & 100.0 & 100.0 \\
\hline
\end{tabular}

Source: Bank of Algeria.

1/ Conversions of banks' claims on public enterprises to claims on the government results, other things being equal, in a decrease in credit to the economy. 
Table 28. Algeria: Distribution of Credit to the Economy by Sector, 2006-10

\begin{tabular}{lrrrrr}
\hline & 2006 & 2007 & 2008 & 2009 & 2010 \\
\hline & \multicolumn{5}{c}{ (In billions of dinars; end of period) } \\
Public sector & 847.0 & 988.9 & $1,201.9$ & $1,485.1$ & $1,460.6$ \\
Private sector & $1,057.0$ & 1216 & $1,413.3$ & $1,600.6$ & $1,806.7$ \\
Local administration & 1.4 & 0.3 & 0.3 & 0.8 & 0.8 \\
Total & $1,905.4$ & $2,205.2$ & $2,615.5$ & $3,086.5$ & $3,268.1$ \\
& & (Annual percentage change) & \\
Public sector & -4.0 & 16.8 & 21.5 & 23.6 & -1.6 \\
Private sector & 17.8 & 15.0 & 16.2 & 13.3 & 12.9 \\
Local administration & $1,300.0$ & -78.6 & 0.0 & 166.7 & 0.0 \\
Total & 7.1 & 15.7 & 18.6 & 18.0 & 5.9 \\
& & $($ In percent of total credits) & \\
Public sector & 44.5 & 44.8 & 46.0 & 48.1 & 44.7 \\
Private sector & 55.5 & 55.1 & 54.0 & 51.9 & 55.3 \\
Local administration & 0.1 & 0.0 & 0.0 & 0.0 & 0.0 \\
Total & 100.0 & 100.0 & 100.0 & 100.0 & 100.0 \\
\hline
\end{tabular}

Source: Bank of Algeria. 
Table 29. Algeria: Structure of Interest Rates, 2006-10

(In percent per annum)

\begin{tabular}{|c|c|c|c|c|c|}
\hline & \multicolumn{5}{|c|}{ December } \\
\hline & 2006 & 2007 & 2008 & 2009 & 2010 \\
\hline Central bank deposit auction $1 /$ & 1.86 & 1.92 & 1.44 & $\ldots$ & $\ldots$ \\
\hline Central bank rediscount & 4.0 & 4.0 & 4 & 4.0 & 4.0 \\
\hline Central bank overdraft & 19.0 & 19.0 & 19.0 & 19.0 & 19.0 \\
\hline \multicolumn{6}{|l|}{ Money market } \\
\hline Repurchase agreements & $\ldots$ & $\ldots$ & $\ldots$ & $\ldots$ & $\ldots$ \\
\hline Central bank credit auction & $\ldots$ & $\ldots$ & $\ldots$ & $\ldots$ & $\ldots$ \\
\hline Commercial banks' deposit rates & $1.25-2.50$ & $1.25-2.50$ & $1.25-2.50$ & $1.25-2.50$ & $1.25-2.50$ \\
\hline Commercial banks' lending rates & $5.50-9.00$ & $5.50-9.00$ & $5.50-9.00$ & $5.50-9.00$ & $5.50-9.00$ \\
\hline \multicolumn{6}{|l|}{ CNEP (savings and housing bank) } \\
\hline \multicolumn{6}{|l|}{ Deposit rates } \\
\hline Savings accounts & $2.0-5.0$ & $2.0-5.0$ & $2.0-5.1$ & $2.0-2.5$ & $2.0-2.5$ \\
\hline Housing accounts & 5.0 & 5.0 & 5.0 & 5.0 & 5.0 \\
\hline \multicolumn{6}{|l|}{ Lending rates (housing) } \\
\hline Individuals & $5.75-7.50$ & $5.50-5.75$ & $5.50-5.75$ & $5.50-5.75$ & $5.50-5.76$ \\
\hline Developers & $0.7-2.0$ & $0.7-2.0$ & $0.7-2.0$ & $0.7-2.0$ & $0.7-2.0$ \\
\hline \multicolumn{6}{|l|}{ Treasury securities } \\
\hline 13 weeks & 1.44 & 0.20 & 0.18 & 0.32 & 0.18 \\
\hline 26 weeks & 1.65 & 0.33 & 0.19 & 0.35 & 0.21 \\
\hline 52 weeks & 2.38 & 2.60 & $\ldots$ & $\ldots$ & $\ldots$ \\
\hline 2 years & 2.81 & 2.90 & $\ldots$ & $\ldots$ & $\ldots$ \\
\hline 5 years & 3.16 & 3.25 & $\ldots$ & $\ldots$ & $\ldots$ \\
\hline 7 years & 4.00 & 4.00 & $\ldots$ & $\ldots$ & $\ldots$ \\
\hline 10 years & 4.26 & 4.50 & $\ldots$ & $\ldots$ & $\ldots$ \\
\hline \multicolumn{6}{|l|}{ Memorandum item: } \\
\hline $\begin{array}{l}\text { Consumer price index (average } \\
\text { percentage change) }\end{array}$ & 2.5 & 3.5 & 4.5 & 5.7 & 3.9 \\
\hline
\end{tabular}

Source: Algerian authorities.

1/ One-week tender rate for 2002-04; weighted average of one- and three-month tender rates thereafter. 
Table 30. Algeria: Number of Branches of Financial Institutions, 2006-10

\begin{tabular}{|c|c|c|c|c|c|}
\hline & 2006 & 2007 & 2008 & 2009 & 2010 \\
\hline Bank of Algeria & 49 & 49 & 49 & 49 & 49 \\
\hline \multicolumn{6}{|l|}{ Banque de l'Agriculture et du Développement } \\
\hline Rural (BADR) & 291 & 291 & 290 & 290 & 290 \\
\hline Banque de Développement Local (BDL) & 146 & 146 & 147 & 148 & 149 \\
\hline Banque Extérieure d'Algérie (BEA) & 84 & 84 & 86 & 91 & 91 \\
\hline Banque Nationale d'Algérie (BNA) & 176 & 184 & 189 & 197 & 199 \\
\hline Crédit Populaire d'Algérie (CPA) & 132 & 133 & 136 & 137 & 139 \\
\hline Caisse Nationale d'Epargne et de Prévoyance (CNEP) & 201 & 206 & 209 & 209 & 209 \\
\hline Société de Refinancement Hypothécaire (SRH) & 1 & 1 & 1 & 1 & 1 \\
\hline Caisse Nationale de Mutualité Agricole (CNMA) & 62 & 62 & 62 & 62 & 62 \\
\hline Union Bank & 0 & 0 & 0 & 0 & 0 \\
\hline El-Khalifa Bank & 0 & 0 & 0 & 0 & 0 \\
\hline Mouna Bank & 0 & 0 & 0 & 0 & 0 \\
\hline Banque Commerciale et Industrielle d'Algérie (BCIA) & 0 & 0 & 0 & 0 & 0 \\
\hline Algerian International Bank & 0 & 0 & 0 & 0 & 0 \\
\hline Arcobank & 0 & 0 & 0 & 0 & 0 \\
\hline Compagnie Algérienne de Banque (CAB) & 0 & 0 & 0 & 0 & 0 \\
\hline Bank El Baraka & 16 & 17 & 20 & 20 & 21 \\
\hline Citibank & 4 & 4 & 4 & 4 & 4 \\
\hline Arab Banking Corporation (ABC Algeria) & 6 & 7 & 10 & 12 & 13 \\
\hline Société Générale & 24 & 37 & 53 & 54 & 63 \\
\hline Natexis El Amana Bank & 4 & 10 & 12 & 12 & 13 \\
\hline Al Rayan Algerian Bank & 0 & 0 & 0 & 0 & 0 \\
\hline Arab Bank & 2 & 4 & 4 & 4 & 4 \\
\hline BNP Paribas El-Djazair & 18 & 36 & 46 & 49 & 55 \\
\hline Banque Générale Méditerranéenne & 0 & 0 & 0 & 0 & 0 \\
\hline TRUST Bank—Algeria & 3 & 3 & 6 & 7 & 11 \\
\hline The Housing Bank for Trade and Finance_-Algeria & 2 & 2 & 4 & 4 & 4 \\
\hline Gulf Bank-Algeria & 1 & 1 & 13 & 13 & 21 \\
\hline Sofinance & 1 & 1 & 1 & 1 & 1 \\
\hline Arab Leasing Corporation & 2 & 3 & 3 & 3 & 3 \\
\hline Cetelem & 1 & 1 & 1 & 1 & 1 \\
\hline Fransabank el dzajair & 0 & 1 & 1 & 1 & 2 \\
\hline Calyon Algeria & 0 & 1 & 1 & 1 & 1 \\
\hline HSBC -EI Djazair & 0 & 1 & 1 & 1 & 2 \\
\hline Al Salem Bank & 0 & 1 & 1 & 2 & 2 \\
\hline Société algérienne de leasing immobilier & 1 & 1 & 0 & 0 & 0 \\
\hline Maghreb Leasing Algerie & 1 & 1 & 1 & 1 & 2 \\
\hline Total & 1,228 & 1,288 & 1,351 & 1,374 & 1,412 \\
\hline
\end{tabular}

Source: Bank of Algeria. 
Table 31. Algeria: Balance of Payments, 2006-10

(In billions of U.S. dollars; unless otherwise indicated)

\begin{tabular}{|c|c|c|c|c|c|}
\hline & 2006 & 2007 & 2008 & 2009 & 2010 \\
\hline Current account & 29.0 & 30.6 & 34.5 & 0.4 & 12.2 \\
\hline Trade balance & 34.1 & 34.2 & 40.6 & 7.8 & 18.2 \\
\hline Exports, f.o.b. & 54.7 & 60.6 & 78.6 & 45.2 & 57.1 \\
\hline Hydrocarbons & 53.6 & 59.6 & 77.2 & 44.4 & 56.1 \\
\hline Other exports & 1.1 & 1.0 & 1.4 & 0.8 & 1.0 \\
\hline Imports, f.o.b. & -20.7 & -26.4 & -38.0 & -37.4 & -38.9 \\
\hline Services and income (net) & -6.7 & -5.9 & -8.9 & -10.0 & -8.7 \\
\hline Services (net) & -2.2 & -4.0 & -7.6 & -8.7 & -8.3 \\
\hline Credit & 2.6 & 2.9 & 3.5 & 3.0 & 3.6 \\
\hline Debit & -4.8 & -6.9 & -11.1 & -11.7 & -11.9 \\
\hline Income (net) & -4.5 & -1.8 & -1.3 & -1.3 & -0.4 \\
\hline Credit & 2.4 & 3.8 & 5.1 & 4.7 & 4.6 \\
\hline Debit & -6.9 & -5.6 & -6.5 & -6.1 & -5.0 \\
\hline \multicolumn{6}{|l|}{ Of which: } \\
\hline Interest payments & -0.8 & -0.2 & -0.2 & -0.2 & -0.1 \\
\hline Profit repatriation & -6.2 & -5.4 & -4.6 & -5.9 & -4.9 \\
\hline Transfers (net) & 1.6 & 2.2 & 2.8 & -3.9 & -4.0 \\
\hline Capital account & -11.2 & -1.1 & 2.5 & 3.5 & 3.4 \\
\hline Direct investment (net) & 1.8 & 1.4 & 2.3 & 2.5 & 3.5 \\
\hline Loans (net) & -11.9 & -0.8 & -0.4 & 1.5 & 0.1 \\
\hline Drawings & 1.0 & 0.5 & 0.8 & 2.2 & 0.6 \\
\hline Amortization & -12.9 & -1.3 & -1.3 & -0.7 & -0.4 \\
\hline Short-term capital and errors and omissions & -1.1 & -1.7 & 0.6 & -0.6 & -0.2 \\
\hline Overall balance & 17.7 & 29.6 & 37.0 & 3.9 & 15.6 \\
\hline Financing & -17.7 & -29.6 & -37.0 & 3.9 & 15.58 \\
\hline Official reserves (increases -) & -17.7 & -29.6 & -37.0 & -3.9 & -15.58 \\
\hline Fund repurchases & 0.0 & 0.0 & 0.0 & 0.0 & 0.0 \\
\hline \multicolumn{6}{|l|}{ Memorandum items: } \\
\hline Current account balance (in percent of GDP) & 24.7 & 22.5 & 20.1 & 0.3 & 7.6 \\
\hline Algerian crude oil export unit value (US $\$ /$ barrel) 1/ & 65.7 & 74.7 & 100.0 & 62.3 & 80.15 \\
\hline Gross official reserves (in billions of US\$) & 77.8 & 110.2 & 143.1 & 148.9 & 162.2 \\
\hline In months of next years' imports of goods and service! & 28.0 & 27.4 & 35.2 & 36.4 & 38.3 \\
\hline Gross external debt (in billions of US\$) & 5.6 & 5.1 & 5.6 & 5.7 & 5.7 \\
\hline External debt/exports (in percent) & 10.2 & 8.4 & 6.8 & 10.9 & 10.0 \\
\hline External debt/GDP (in percent) & 4.8 & 3.8 & 3.3 & 3.8 & 3.5 \\
\hline Debt service (in billions of US\$) & 13.6 & 1.5 & 1.5 & 1.1 & 0.5 \\
\hline Debt service/exports (in percent) & 24.9 & 2.5 & 1.8 & 2.2 & 0.9 \\
\hline Interest payments/exports (in percent) & 1.4 & 0.4 & 0.2 & 0.4 & 0.2 \\
\hline GDP (in billions of US\$) & 116.8 & 135.3 & 171.0 & 139.8 & 160.8 \\
\hline
\end{tabular}

Sources: Algerian authorities; and Fund staff estimates.

$1 /$ Weighted average of quarterly data. 
Table 32. Algeria: Exports and Imports, 2006-10

\begin{tabular}{|c|c|c|c|c|c|}
\hline & 2006 & 2007 & 2008 & 2009 & 2010 \\
\hline & \multicolumn{5}{|c|}{ (In millions of U.S. dollars) } \\
\hline \multicolumn{6}{|l|}{ Exports } \\
\hline Food & 73 & 88 & 122 & 113 & 306 \\
\hline Hydrocarbon & 53,608 & 59,605 & 77,195 & 44,415 & 56,121 \\
\hline Raw materials & 195 & 170 & 339 & 169 & 165 \\
\hline Semi finished & 765 & 640 & 853 & 393 & 434 \\
\hline Agricultural equipment & 1 & 1 & 1 & 0 & 1 \\
\hline Industrial equipment & 44 & 46 & 77 & 42 & 27 \\
\hline Consumer goods & 44 & 35 & 35 & 49 & 34 \\
\hline Other & 10 & 5 & 8 & 5 & 2 \\
\hline Total & 54,740 & 60,590 & 78,630 & 45,186 & 57,090 \\
\hline Of which: nonhydrocarbon & 1,132 & 985 & 1,426 & 766 & 969 \\
\hline \multicolumn{6}{|l|}{ Imports } \\
\hline Food & 3,572 & 4,656 & 7,273 & 5,512 & 5,726 \\
\hline Energy & 230 & 305 & 1,722 & 516 & 888 \\
\hline Raw materials & 792 & 1,245 & 133 & 1,128 & 1,335 \\
\hline Semi finished & 4,637 & 6,678 & 9,251 & 9,557 & 9,446 \\
\hline Agricultural equipment & 90 & 137 & 161 & 219 & 325 \\
\hline Industrial equipment & 8,015 & 9,361 & 14,096 & 14,141 & 14,794 \\
\hline Consumer goods & 2,830 & 3,546 & 4,125 & 5,868 & 5,687 \\
\hline Direct investment in kind & 515 & 420 & 631 & 462 & 684 \\
\hline \multirow[t]{2}{*}{ Total } & 20,681 & 26,348 & 37,391 & 37,403 & 38,885 \\
\hline & \multicolumn{5}{|c|}{ (In percent) } \\
\hline \multicolumn{6}{|l|}{ As share of total exports } \\
\hline Nonhydrocarbon & 2.1 & 1.6 & 1.8 & 1.7 & 1.7 \\
\hline \multicolumn{6}{|l|}{ As share of total imports } \\
\hline Food as share of imports & 17.3 & 17.7 & 19.5 & 14.7 & 14.7 \\
\hline Industrial goods & 38.8 & 35.5 & 37.7 & 37.8 & 38.0 \\
\hline Consumer goods & 13.7 & 13.5 & 11.0 & 15.7 & 14.6 \\
\hline
\end{tabular}

Source: Algerian authorities. 
Table 33. Algeria: Volume of Hydrocarbon Exports, 2006-10

\begin{tabular}{lrrrrr}
\hline & 2006 & 2007 & 2008 & 2009 & 2010 \\
\hline & & (In billions of BTUs) & \\
Liquid petroleum products & & & & & \\
$\quad$ Crude oil & 1,735 & 1,708 & $1,545.7$ & $1,371.0$ & $1,301.6$ \\
$\quad$ Condensate & 598 & 576 & 580.9 & 467.4 & 271.6 \\
$\quad$ Refined petroleum products & 492 & 432 & 456.0 & 473.0 & 618.4 \\
LPG & 295 & 337 & 338.0 & 307.7 & 267.2 \\
Total liquid petroleum & 3,120 & 3,053 & $2,920.6$ & $2,619.1$ & $2,458.8$ \\
Natural gas piped and LNG & & & & & \\
$\quad$ Natural gas piped & 1,416 & 1,326 & $1,462.8$ & $1,250.6$ & 732.2 \\
LNG & 912 & 934 & 811.6 & 791.8 & $1,415.2$ \\
Total gas & 2,328 & 2,260 & $2,274.4$ & $2,042.4$ & $2,147.4$ \\
Total hydrocarbon & 5,448 & 5,313 & $5,195.0$ & $4,661.5$ & $4,606.2$ \\
& $($ In percent of total hydrocarbon volume) & \\
Liquid petroleum products & & & & & \\
$\quad$ Crude oil & 31.9 & 32.1 & 29.8 & 29.4 & 28.3 \\
Condensate & 11.0 & 10.8 & 11.2 & 10.0 & 5.9 \\
Refined petroleum products & 9.0 & 8.1 & 8.8 & 10.1 & 13.4 \\
LPG & 5.4 & 6.3 & 6.5 & 6.6 & 5.8 \\
Total & 57.3 & 57.5 & 56.2 & 56.2 & 53.4 \\
Natural gas and LNG & & & & & \\
$\quad$ Natural gas & & & & & \\
LNG & 26.0 & 25.0 & 28.2 & 26.8 & 15.9 \\
Total & 16.7 & 17.6 & 15.6 & 17.0 & 30.7 \\
\hline & 42.7 & 42.5 & 43.8 & 43.8 & 46.6 \\
\hline
\end{tabular}

Source: Algerian authorities. 
Table 34. Algeria: Hydrocarbon Exports, 2006-10

\begin{tabular}{|c|c|c|c|c|c|}
\hline & 2006 & 2007 & 2008 & 2009 & 2010 \\
\hline \multicolumn{6}{|l|}{ Crude oil } \\
\hline Value (in millions of US\$) & 22,697 & 25,374 & 30,514 & 16,856 & 20,725 \\
\hline Of which: Profit repatriation of partners & 4,625 & 2,989 & 3,593 & 2,963 & 3,117 \\
\hline Volume (in millions of barrels) & 345.3 & 339.5 & 305.5 & 272.8 & 258.7 \\
\hline Unit price (in US\$ per barrel) & 65.7 & 74.7 & 99.9 & 62.2 & 80.2 \\
\hline \multicolumn{6}{|l|}{ Condensate } \\
\hline Value (in millions of US\$) & 7,268 & 8,498 & 10,090 & 5,146 & 4,269 \\
\hline Of which: Profit repatriation of partners & 153 & 228 & 221 & 232 & 196 \\
\hline Volume (in millions of barrels) & 119.1 & 114.9 & 115.8 & 93.3 & 54.3 \\
\hline Unit price (in US\$ per barrel) & 61.0 & 73.9 & 87.2 & 55.2 & 79 \\
\hline \multicolumn{6}{|l|}{ Refined petroleum products } \\
\hline Value (in millions of US\$) & 4,756 & 5,838 & 7310 & 5,373 & $9,219.5$ \\
\hline Volume (in millions of barrels) & 76 & 78 & 82.3 & 85.4 & 111.6 \\
\hline Unit price (in US\$ per barrel) & 62.8 & 75.3 & 88.8 & 62.9 & 82.6 \\
\hline \multicolumn{6}{|l|}{ LPG } \\
\hline Value (in millions of US\$) & 3,626 & 4,545 & 5,708 & 3,217 & 4,087 \\
\hline Of which: Profit repatriation of partners & 239 & 367 & 365 & 469 & 373 \\
\hline Volume (in millions of barrels) & 82.0 & 87.3 & 81.5 & 74.1 & 64.3 \\
\hline Unit price (in US\$ per barrel) & 44.2 & 52.1 & 70.0 & 43.4 & 63.5 \\
\hline \multicolumn{6}{|l|}{ LNG } \\
\hline Value (in millions of US\$) & 6,383 & 6,725 & 9,092 & 5,598 & 5,879 \\
\hline Volume (in billions of c.m. dry gas equivalent) & 24.0 & 24.1 & 34.6 & 34.7 & 31.2 \\
\hline Unit price (in US\$ per thousand c.m.) & 266.4 & 279.6 & 262.7 & 161.3 & 188.6 \\
\hline \multicolumn{6}{|l|}{ Natural gas piped (NG) } \\
\hline Value (in millions of US\$) & 8,877 & 8,625 & 14,481 & 8,225 & 11,942 \\
\hline Of which: Profit repatriation of partners & 271 & 311 & 383 & 258 & 251 \\
\hline Volume (in billions of c.m.) & 37.9 & 35.4 & 39.0 & 33.6 & 37.8 \\
\hline Unit price (in US\$ per thousand c.m.) & 234.5 & 243.8 & 371.3 & 244.8 & 311.6 \\
\hline Total hydrocarbon receipts (in million of US\$) & 53,608 & 59,605 & 77,195 & 44,415 & 56,122 \\
\hline Of which: Share of Sonatrach's partners & 5,288 & 3,895 & 4,562 & 3,921 & 3,938 \\
\hline Oil products & 71.5 & 74.2 & 69.5 & 68.9 & 68.2 \\
\hline Crude oil & 42.3 & 42.6 & 39.5 & 38.0 & 36.9 \\
\hline Condensate & 13.6 & 14.3 & 13.1 & 11.6 & 7.6 \\
\hline Refined petroleum products & 8.9 & 9.8 & 9.5 & 12.1 & 16.4 \\
\hline LPG & 6.8 & 7.6 & 7.4 & 7.2 & 7.3 \\
\hline Gas & 28.5 & 25.8 & 30.5 & 31.1 & 31.8 \\
\hline LNG & 11.9 & 11.3 & 11.8 & 12.6 & 10.5 \\
\hline NG & 16.6 & 14.5 & 18.8 & 18.5 & 21.3 \\
\hline
\end{tabular}

Source: Algerian authorities. 
Table 35. Algeria: Trade Indices, 2005-09

\begin{tabular}{lccccc}
\hline & 2006 & 2007 & 2008 & 2009 & 2010 \\
\hline & \multicolumn{7}{c}{$(1995=100)$} \\
Total export volume index & 267.6 & 264.0 & 256.7 & 229.2 & 223.7 \\
Nonhydrocarbon export volume index & 265.7 & 208.2 & 274.4 & 168.4 & 208.9 \\
Total import volume index & 135.3 & 154.2 & 175.8 & 200.4 & 228.5 \\
Food import volume index & 150.4 & 173.1 & 234.6 & 209.2 & 193.4 \\
Terms of trade index & 324.3 & 326.7 & 400.3 & 293.0 & 360.0 \\
& & $($ Annual percentage change) & \\
Total export volume index & -1.7 & -1.3 & -2.8 & -10.7 & -2.4 \\
Nonhydrocarbon export volume index & 47.9 & -21.7 & 31.8 & -38.6 & 24.0 \\
Total import volume index & -5.7 & 14.0 & 14.0 & 14.0 & 14.0 \\
Food import volume index & -4.6 & 15.1 & 35.5 & -10.8 & -7.6 \\
Terms of trade index & 9.6 & 0.8 & 22.5 & -26.8 & 22.9 \\
\hline
\end{tabular}

Sources: Algerian authorities; and Fund staff calculations. 
Table 36. Algeria: External Debt, 2006-101/

(In millions of U.S. dollars; unless otherwise indicated)

\begin{tabular}{|c|c|c|c|c|c|}
\hline & 2006 & 2007 & 2008 & 2009 & 2010 \\
\hline Total external debt & 5,674 & 5,606 & 5,586 & 5,413 & 5,164 \\
\hline Long-term debt & 5,124 & 4,889 & 4,282 & 3,921 & 3,386 \\
\hline Official creditors & 2,281 & 2401 & 2,073 & 2,141 & 2,855 \\
\hline Multilateral (excluding IMF) & 402 & 226 & 10 & 10 & 9 \\
\hline Of which: World Bank & 119 & 113 & 0 & 0 & 0.52 \\
\hline Bilateral & 1,879 & 2,175 & 2,063 & 2,131 & 2,846 \\
\hline Concessional & 1,535 & 1,777 & 1,685 & 1,741 & 2,325 \\
\hline Private creditors & 2,843 & 2,488 & 2,209 & 1,780 & 531 \\
\hline Commercial banks & 29 & 49 & 88 & 73 & 50 \\
\hline Supplier 2/ & 92 & 16 & 6 & 21 & 10 \\
\hline Other private & 2,722 & 2,423 & 2,115 & 1,686 & 471 \\
\hline IMF & 0 & 0 & 0 & 0 & 0 \\
\hline Short-term debt & 550 & 717 & 1,304 & 1,492 & 1,778 \\
\hline Disbursements & & 339 & 406 & 199 & 56 \\
\hline Official creditors & 126 & 196 & 179 & 81 & 55 \\
\hline Multilateral (excluding IMF) & 67 & 108 & 3 & 0 & 0 \\
\hline Of which: World Bank & $\ldots$ & 9 & 3 & 0 & 0 \\
\hline Bilateral & 59 & 88 & 176 & 81 & 55 \\
\hline Private creditors & 75 & 143 & 227 & 118 & 2 \\
\hline IMF purchases & 0 & 0 & 0 & 0 & 0 \\
\hline Principal repayment & 12,726 & 976 & 1,067 & 875 & 483 \\
\hline Official creditors & 10,851 & 228 & 914 & 437 & 151 \\
\hline Multilateral (excluding IMF) & 2,340 & 30 & 104 & 2 & 1 \\
\hline Of which: World Bank & 677 & 17 & 104 & 1 & 1 \\
\hline Bilateral & 8,511 & 199 & 810 & 435 & 150 \\
\hline Private creditors & 1,723 & 412 & 153 & 438 & 256 \\
\hline IMF repurchases & 0 & 0 & 0 & 0 & 0 \\
\hline Interest payments & 610 & 200 & 151 & 125 & 105 \\
\hline Official creditors & 435 & 62 & 109 & 0 & 31 \\
\hline Multilateral (excluding IMF) & 118 & 13 & 2 & 0 & 0 \\
\hline Of which: World Bank & 21 & 7 & 2 & 0 & 0 \\
\hline Bilateral & 316 & 48 & 107 & 84 & 30 \\
\hline Private creditors & 122 & 73 & 42 & 41 & 71 \\
\hline IMF charges & 1 & 0 & 0 & 0 & 0 \\
\hline Short-term debt & $\cdots$ & $\cdots$ & $\cdots$ & $\cdots$ & $\ldots$ \\
\hline Total debt service & 13,351 & 1,175 & 1,218 & 1,000 & 509 \\
\hline Official creditors & 11,286 & 290 & 1,023 & 522 & 181 \\
\hline Multilateral (excluding IMF) & 2,458 & 43 & 107 & 2 & 1 \\
\hline Bilateral & 8,828 & 247 & 916 & 519 & 180 \\
\hline Private creditors & 1,845 & 485 & 195 & 478 & 327 \\
\hline IMF repurchases and charges & 1 & 0 & 0 & 0 & 0 \\
\hline Short-term debt & 15 & 0 & 0 & 0 & 0 \\
\hline
\end{tabular}

Sources: Algerian authorities; and Fund staff calculations. 
Table 37. Nominal and Real Effective Exchange Rates, 1990-2010

$(2005=100)$

\begin{tabular}{|c|c|c|c|c|}
\hline & Q1 & Q2 & Q3 & Q4 \\
\hline \multicolumn{5}{|c|}{ Nominal effective exchange rate } \\
\hline 1990 & 722.7 & 693.3 & 599.6 & 500.1 \\
\hline 1991 & 341.9 & 329.3 & 329.1 & 253.0 \\
\hline 1992 & 253.5 & 254.4 & 248.7 & 257.0 \\
\hline 1993 & 262.4 & 260.4 & 262.5 & 265.0 \\
\hline 1994 & 266.8 & 176.6 & 157.0 & 144.0 \\
\hline 1995 & 137.9 & 125.1 & 114.8 & 113.5 \\
\hline 1996 & 111.1 & 110.8 & 109.1 & 109.6 \\
\hline 1997 & 114.6 & 117.7 & 119.3 & 121.2 \\
\hline 1998 & 124.4 & 124.6 & 124.1 & 122.0 \\
\hline 1999 & 117.5 & 115.5 & 117.4 & 117.2 \\
\hline 2000 & 116.9 & 116.9 & 115.6 & 116.5 \\
\hline 2001 & 118.1 & 121.0 & 120.7 & 121.7 \\
\hline 2002 & 121.1 & 115.2 & 109.4 & 108.7 \\
\hline 2003 & 104.6 & 100.6 & 103.0 & 105.9 \\
\hline 2004 & 103.4 & 106.4 & 104.2 & 98.8 \\
\hline 2005 & 97.5 & 99.0 & 101.0 & 102.5 \\
\hline 2006 & 101.7 & 99.8 & 99.1 & 99.8 \\
\hline 2007 & 99.2 & 97.8 & 99.4 & 97.0 \\
\hline 2008 & 95.8 & 97.3 & 104.3 & 106.3 \\
\hline 2009 & 100.9 & 96.6 & 92.9 & 91.1 \\
\hline \multirow[t]{2}{*}{2010} & 93.8 & 97.0 & 94.8 & 92.5 \\
\hline & \multicolumn{3}{|c|}{ Real effective exchange rate } & \\
\hline 1990 & 240.6 & 245.2 & 216.2 & 191.0 \\
\hline 1991 & 137.2 & 136.8 & 141.7 & 117.1 \\
\hline 1992 & 123.3 & 134.0 & 141.0 & 148.5 \\
\hline 1993 & 156.9 & 162.3 & 165.9 & 172.7 \\
\hline 1994 & 185.8 & 131.5 & 126.0 & 123.7 \\
\hline 1995 & 126.3 & 118.7 & 114.4 & 115.6 \\
\hline 1996 & 118.1 & 122.5 & 121.5 & 122.4 \\
\hline 1997 & 127.2 & 129.3 & 132.0 & 134.5 \\
\hline 1998 & 137.9 & 137.8 & 137.9 & 135.0 \\
\hline 1999 & 128.5 & 126.1 & 127.2 & 125.3 \\
\hline 2000 & 124.4 & 121.6 & 118.8 & 118.7 \\
\hline 2001 & 120.6 & 125.4 & 125.2 & 127.0 \\
\hline 2002 & 125.1 & 116.3 & 110.1 & 108.7 \\
\hline 2003 & 103.1 & 101.1 & 104.4 & 107.8 \\
\hline 2004 & 104.9 & 107.6 & 105.4 & 99.9 \\
\hline 2005 & 99.3 & 100.3 & 99.7 & 100.7 \\
\hline 2006 & 101.1 & 99.9 & 98.1 & 100.0 \\
\hline 2007 & 98.8 & 97.4 & 100.2 & 97.4 \\
\hline 2008 & 97.0 & 99.1 & 106.6 & 110.2 \\
\hline 2009 & 106.4 & 102.4 & 100.4 & 98.7 \\
\hline 2010 & 100.9 & 105.2 & 103.4 & 100.2 \\
\hline
\end{tabular}

Source: International Monetary Fund.

CInternational Monetary Fund. Not for Redistribution 